Acta Crystallographica Section A

Foundations of

Crystallography

ISSN 0108-7673

Received 22 September 2005

Accepted 2 December 2005

(C) 2006 International Union of Crystallography Printed in Great Britain - all rights reserved

\section{Bilbao Crystallographic Server. II. Representations of crystallographic point groups and space groups}

\author{
Mois I. Aroyo, ${ }^{a *}$ Asen Kirov, ${ }^{b}$ Cesar Capillas, ${ }^{a}$ J. M. Perez-Mato ${ }^{a}$ and Hans \\ Wondratschek $^{\mathrm{c}}$
}

\begin{abstract}
${ }^{a}$ Departamento de Física de la Materia Condensada, Universidad del País Vasco, E-48080 Bilbao, Spain, ${ }^{\mathbf{b}}$ Condensed Matter Physics Department, Sofia University, BG-1486 Sofia, Bulgaria, and

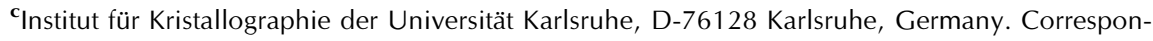
dence e-mail:wmpararm@|g.ehu.es
\end{abstract}

\begin{abstract}
The Bilbao Crystallographic Server is a web site with crystallographic programs and databases freely available on-line (http://www.cryst.ehu.es). The server gives access to general information related to crystallographic symmetry groups (generators, general and special positions, maximal subgroups, Brillouin zones etc.). Apart from the simple tools for retrieving the stored data, there are programs for the analysis of group-subgroup relations between space groups (subgroups and supergroups, Wyckoff-position splitting schemes etc.). There are also software packages studying specific problems of solid-state physics, structural chemistry and crystallography. This article reports on the programs treating representations of point and space groups. There are tools for the construction of irreducible representations, for the study of the correlations between representations of group-subgroup pairs of space groups and for the decompositions of Kronecker products of representations.
\end{abstract}

\section{Introduction}

The Bilbao Crystallographic Server is a web site with crystallographic databases and programs that can be used free of charge from any web browser via the Internet. The server has been on-line for more than 6 years and new programs are continuously added to the available tools (Kroumova et al., 2003). The server gives access to data in International Tables for Crystallography (2002), Volume A: Space Group Symmetry (abbreviated as ITA) and data of maximal subgroups of plane and space groups as listed in International Tables for Crystallography (2004), Volume A1: Symmetry Relations between Space Groups (abbreviated as ITA1). We have also started developing a database for subperiodic groups: the basic crystallographic data of the layer and rod groups [International Tables for Crystallography (2002), Volume E: Subperiodic Groups (abbreviated as ITE)] and their maximal subgroups are already accessible on the server. The server includes the so-called $\mathbf{k}$-vector or Brillouin-zone database. It consists of Brillouin-zone figures and tables for all 230 space groups of the wavevector symmetry types, which are fundamental for the classification of the space-group irreducible representations (abbreviated as irreps). A database on incommensurate structures, still under development, that includes modulated structures and composites is also available.

The accompanying software is divided into several shells according to its complexity and proximity to the information contained in the database kernel. There are simple tools for retrieving data directly from the database such as generators and general positions, Wyckoff-position data and maximal subgroups. In addition, we have developed different applications that are essential for problems involving group-subgroup relations between space groups: subgroups and supergroups of space groups, graphs of maximal subgroups for an arbitrary group-subgroup pair, Wyckoffposition splitting schemes for group-subgroup pairs etc. In a recent publication, we have provided a detailed description of the set of databases available on the server and the shell formed by the crystallographic computing programs (Aroyo et al., 2006).

The aim of this contribution is to report on the development of a shell with programs facilitating the application of representation theory to specific problems of solid-state physics and crystallography-related fields. The computing packages support certain essential (and more involved from a mathematical point of view) steps in the related group-theoretical studies. The server offers access to the basic modules for handling space-group (REPRES) and point-group (POINT) representations, it enables the study of the correlations between irreps of group-subgroup-related space groups (CORREL) and the decomposition of Kronecker direct products of space-group irreps (DIRPRO). In the following, the group-theoretical background of the developed programs is outlined. Illustrative examples explain the necessary input data and provide details on the output results. 


\section{Representations of crystallographic groups}

\subsection{Space-group representations}

2.1.1. The problem. There exist several reference sets of tables of space-group irreps [see e.g. Cracknell et al. (1979), hereafter referred to as CDML, and the references therein]. However, the available data have important drawbacks related to the lack of full space-group representations owing to the limitations and/or specificity in the choice of the $\mathbf{k}$ vectors. In addition, the space-group settings used are often not compatible with those of ITA. These disadvantages are overcome by the program REPRES which computes the irreps of space groups explicitly. For any space group $\mathcal{G}$ and a $\mathbf{k}$ vector, the corresponding little group $\mathcal{G}^{\mathbf{k}}$, the allowed (littlegroup) irreps and the matrices of the full-group irreps are constructed. As part of the working environment of the Bilbao Crystallographic Server, the program REPRES provides the irrep data in a format suitable for its further use as input for other programs on the server.

2.1.2. The method. REPRES calculates the irreps of space groups following the algorithm applied in KAREP (Hovestreydt et al., 1992). Both programs apply a general scheme based on a normal-subgroup induction method of constructing the irreps of a group $\mathcal{G}$ starting from those of a normal subgroup $\mathcal{H} \triangleleft \mathcal{G}$. The main steps of the procedure involve the construction of all irreps of $\mathcal{H}$ and their distribution into orbits under $\mathcal{G}$, determination of the corresponding little groups and the allowed (small) irreps and, finally, construction of the irreps of $\mathcal{G}$ by induction from the allowed irreps. The main difference between the procedures applied in $K A R E P$ and REPRES concerns the algorithm for the computation of the composition series of the little group: the more general procedure used in REPRES allows the calculations of irreps of space groups defined with respect to any basis. (Only a restricted number of unconventional settings of space groups can be treated by $K A R E P$.)

The application of the general normal-subgroup induction procedure in the case of space groups is straightforward. A normal subgroup of every space group is its translation group $\mathcal{T}$. The irreps of $\mathcal{T}$ are well known and their distribution into orbits, the determination of the related little groups and the induction of the space-group irreps follow closely the general scheme. The most involved step in the procedure is the determination of the allowed irreps of the little group. In the majority of books on irreps of space groups, this problem is solved by applying the theory of the so-called projective representations. Here we have preferred another approach for the construction of the allowed irreps, which is a slight modification of an induction procedure originally proposed by Zak (1960). It is based on the fact that all space groups are solvable groups, i.e. for every space group one can construct a composition series $\mathcal{G} \triangleright \mathcal{H}_{1} \triangleright \mathcal{H}_{2} \triangleright \ldots \triangleright \mathcal{T}$ such that all factor groups $\mathcal{H}_{i} / \mathcal{H}_{i+1}$ are cyclic groups of order 2 or 3 .

In the following, we present the main steps of the REPRES algorithm for the calculation of the matrices of the spacegroup irreps. To make the exposition self-consistent, we have included some basic space-group concepts and definitions as given in ITA, Part 8, Introduction to Space-Group Symmetry. For representation theory, we have followed the terminology of CDML.

\section{Step 1. Space-group information}

The elements of a space group $\mathcal{G}$ are its symmetry operations which are isometries leaving an existing or a possible crystal structure fixed (see ITA). Referred to a coordinate system $\left(O, \mathbf{a}_{1}, \mathbf{a}_{2}, \mathbf{a}_{3}\right)$, consisting of an origin $O$ and a basis $\mathbf{a}_{k}$, the symmetry operations of the space group $\mathcal{G}$ are described by matrix-column pairs $(\boldsymbol{W}, \boldsymbol{w})$.

The set of translations $t_{i}$ of $\mathcal{G}$ forms the translation group $\mathcal{T}(\mathcal{G})$ of $\mathcal{G}$. For each translation, the corresponding vector is defined and the translations of $\mathcal{G}$ are represented by pairs $\left(\boldsymbol{I}, \boldsymbol{t}_{i}\right)$, where $\boldsymbol{I}$ is the $3 \times 3$ unit matrix and $\boldsymbol{t}_{i}$ is the column of coefficients of the translation vector $\mathbf{t}_{i} \in \mathbf{L}$. The set of all translation vectors of $\mathcal{T}(\mathcal{G})$ is called the vector lattice $\mathbf{L}$ of $\mathcal{G}$.

The group $\mathcal{T}(\mathcal{G})$ is an infinite Abelian normal subgroup of $\mathcal{G}$. The cosets of the coset decomposition of the space group $\mathcal{G}$ relative to $\mathcal{T}(\mathcal{G})$,

$$
\mathcal{G}=\mathcal{T}+\left(\boldsymbol{W}_{2}, \boldsymbol{w}_{2}\right) \mathcal{T}+\ldots+\left(\boldsymbol{W}_{p}, \boldsymbol{w}_{p}\right) \mathcal{T},
$$

form the factor group $\mathcal{G} / \mathcal{T}(\mathcal{G})$, which is isomorphic to the point group $\mathcal{P}_{\mathcal{G}}$ of the space group $\mathcal{G}$ (often designated by $\overline{\mathcal{G}}$ in texts on representation theory of space groups).

For its calculations, REPRES needs a file containing the generators of the space group. It is important to note that the program expects the generators to build up the space group from its translation subgroup in the form of a composition series (see ITA, Part 8).

Step 2. Representations of the translation subgroup $\mathcal{T}$ of $\mathcal{G}$

The irreps of a space group $\mathcal{G}$ are obtained by induction from the irreps of $\mathcal{T}(\mathcal{G})$. If the Born-von Karman boundary conditions $\left(\boldsymbol{I}, \boldsymbol{t}_{o i}\right)^{N_{i}}=(\boldsymbol{I}, \boldsymbol{o})$ are assumed to hold [here $N_{i}$ are appropriate large integers and $\boldsymbol{t}_{o i}$ are the columns $(1,0,0)$, $(0,1,0),(0,0,1)$ for $i=1,2,3$, respectively], then the irreps of $\mathcal{T}(\mathcal{G})$ are given by

$$
\Gamma^{\mathbf{k}}(\mathrm{t})=\exp (-i \mathbf{k} \cdot \mathbf{t})
$$

where $\mathrm{t} \in \mathcal{T}(\mathcal{G})$ is the translation and $\mathbf{t}\left(t_{1}, t_{2}, t_{3}\right)$ is the related translation vector. There are $N_{1} N_{2} N_{3}$ different irreps of $\mathcal{T}(\mathcal{G})$, which are distinguished by their vector $\mathbf{k}$ :

$$
\mathbf{k}=\sum_{i=1}^{3} k_{i} \mathbf{a}_{i}^{*}
$$

where $k_{i}=q_{i} / N_{i}, q_{i}=0,1,2, \ldots, N_{i}-1$. The basis $\mathbf{a}_{1}^{*}, \mathbf{a}_{2}^{*}, \mathbf{a}_{3}^{*}$ is called the basis of the reciprocal lattice $\mathbf{L}^{*}$ and it is the dual basis of $\mathbf{a}_{1}, \mathbf{a}_{2}, \mathbf{a}_{3}$ of $\mathbf{L}$. Its vectors $\mathbf{a}_{i}^{*}$ are defined by the relations $\mathbf{a}_{i} \cdot \mathbf{a}_{j}^{*}=2 \pi \delta_{i j}$, where $\delta_{i j}$ is the Kronecker symbol. ${ }^{\mathbf{1}}$

The set of all vectors $\mathbf{k}$ (known as wavevectors) forms a discontinuous array. The wavevectors $\mathbf{k}$ and $\mathbf{k}^{\prime}=\mathbf{k}+\mathbf{K}$, where $\mathbf{K} \in \mathbf{L}^{*}$, describe the same irrep of $\mathcal{T}$. Therefore, to determine all irreps of $\mathcal{T}$ it is necessary to consider only the

\footnotetext{
${ }^{1}$ Crystallographers use for the basis of the reciprocal lattice a different normalization $\mathbf{a}_{i} \cdot \mathbf{a}_{j}^{*}=\delta_{i j}$ and obtain $\Gamma^{\mathbf{k}}(\mathrm{t})=\exp (-2 \pi i \mathbf{k} \cdot \mathbf{t})$ instead of equation (2).
} 
wavevectors of a small region of the reciprocal space, the so-called fundamental region. In most books and articles on irreps of space groups, the first Brillouin zone (or simply Brillouin zone) is chosen as a fundamental region. An alternative choice is the crystallographic unit cell in reciprocal space that corresponds to the unit cell used in crystallography for the description of crystal structures in direct space ( $c f$. Aroyo \& Wondratschek, 1995, for more details).

\section{Step 3. Orbits of irreps of $\mathcal{T}$ and little groups $\mathcal{G}^{\mathbf{k}}$}

The little co-group of a wavevector $\mathbf{k}, \overline{\mathcal{G}}^{\mathbf{k}}$, is a subgroup of the point group $\overline{\mathcal{G}}$. It consists of all matrices $\boldsymbol{W}^{\mathbf{k}} \in \overline{\mathcal{G}}$ that leave the vector $\mathbf{k}$ invariant or change it to an equivalent one, i.e.

$$
\mathbf{k}=\mathbf{k} \boldsymbol{W}^{\mathbf{k}}+\mathbf{K}, \quad \mathbf{K} \in \mathbf{L}^{*} .
$$

The vector $\mathbf{k}$ is called a general $\mathbf{k}$ vector if $\overline{\mathcal{G}}^{\mathbf{k}}=\{\mathcal{I}\}$; otherwise, $\overline{\mathcal{G}}^{\mathbf{k}}>\{\mathcal{I}\}$ and $\mathbf{k}$ is called a special $\mathbf{k}$ vector.

Consider the coset decomposition of $\overline{\mathcal{G}}$ relative to $\overline{\mathcal{G}}^{\mathbf{k}}$. If $\left\{\boldsymbol{W}_{m}\right\}$ is the corresponding set of coset representatives, then the set $* \mathbf{k}=\left\{\mathbf{k} \boldsymbol{W}_{m}+\mathbf{K}\right\}$ is called the star of $\mathbf{k}$ and the vectors $\mathbf{k} \boldsymbol{W}_{m}+\mathbf{K}$ are called the arms of $* \mathbf{k}$. (Here the lattice vector $\mathbf{K}$ is necessary if $\mathbf{k} \boldsymbol{W}_{m}$ is outside the fundamental region.)

An orbit of $\Gamma^{\mathbf{k}}(\mathcal{T})$ relative to $\mathcal{G}$ comprises all irreps $\Gamma^{\mathbf{k}^{\prime}}(\mathcal{T})$ with $\mathbf{k}^{\prime}$ belonging to $* \mathbf{k}$. From the classification of all $\mathbf{k}$ vectors into stars follows the distribution of the irreps of $\mathcal{T}$ into orbits relative to $\mathcal{G}$. Irreps of $\mathcal{T}$ belonging to the same orbit give rise to equivalent irreps of $\mathcal{G}$, i.e. one needs one $\mathbf{k}$ vector per star in order to obtain each irrep of $\mathcal{G}$ exactly once. A simply connected part of the fundamental region that contains exactly one $\mathbf{k}$ vector of each star of $\mathbf{k}$ is called a representation domain. The crystallographic analog of the representation domain in direct space is the asymmetric unit, cf. ITA.

Given a space group $\mathcal{G}$, its translation subgroup $\mathcal{T}$ and an irrep $\Gamma^{\mathbf{k}}(\mathcal{T})$, one can define the little group $\mathcal{G}^{\mathbf{k}}$ of the wavevector $\mathbf{k}$ : it is a space group that consists of all those elements of $\mathcal{G}$ whose rotation parts $\boldsymbol{W}^{\mathbf{k}}$ leave either $\mathbf{k}$ unchanged or invert it into an equivalent vector,

$$
\mathcal{G}^{\mathbf{k}}=\left\{\left(\boldsymbol{W}^{\mathbf{k}}, \boldsymbol{w}^{\mathbf{k}}\right) \in \mathcal{G} \mid \boldsymbol{W}^{\mathbf{k}} \in \overline{\mathcal{G}}^{\mathbf{k}}\right\} .
$$

\section{Step 4. Allowed irreps of $\mathcal{G}^{\mathbf{k}}$}

The irreps of space groups are obtained by induction from the so-called allowed irreps of the little groups $\mathcal{G}^{\mathbf{k}}$ of $\mathbf{k}$. If $\boldsymbol{D}^{\mathbf{k}, i}\left(\mathcal{G}^{\mathbf{k}}\right)$ is an allowed irrep of $\mathcal{G}^{\mathbf{k}}$, then $\boldsymbol{D}^{\mathbf{k}, i}(\boldsymbol{I}, \boldsymbol{t})=\exp (-i \mathbf{k} \mathbf{t}) \boldsymbol{I}$ holds. The matrix $\boldsymbol{I}$ is the identity matrix with $\operatorname{dim}(\boldsymbol{I})=\operatorname{dim}\left(\boldsymbol{D}^{\mathbf{k}, i}\left(\mathcal{G}^{\mathbf{k}}\right)\right)$.

As already noted, REPRES determines the allowed irreps of the little group $\mathcal{G}^{\mathbf{k}}$ making use of the composition series of the little group $\mathcal{G}^{\mathbf{k}}$ :

$$
\mathcal{G}^{\mathbf{k}} \triangleright \mathcal{H}_{1}^{\mathbf{k}} \triangleright \ldots \triangleright \mathcal{H}_{m-1}^{\mathbf{k}} \triangleright \mathcal{H}_{m}^{\mathbf{k}} \triangleright \ldots \triangleright \mathcal{H}_{n}^{\mathbf{k}}=\mathcal{T}\left(\mathcal{G}^{\mathbf{k}}\right)
$$

with $\left|\mathcal{H}_{m-1}^{\mathbf{k}} / \mathcal{H}_{m}^{\mathbf{k}}\right|=2$ or 3 . The (allowed) irreps of $\mathcal{G}^{\mathbf{k}}$ can be obtained from the (allowed) irreps of $\mathcal{T}\left(\mathcal{G}^{\mathbf{k}}\right)$ by applying several times the general induction procedure 'climbing up' the chain of normal subgroups. In this case, the induction is simplified due to the special indices 2 and 3 that appear in the composition series (6). The corresponding induction formulae for the derivation of the irrep matrices of a group from those of a normal subgroup are given in the following.

Consider a group $\mathcal{A}$, a normal subgroup $\mathcal{B}$ of $\mathcal{A}$ and its irreps $\boldsymbol{D}(\mathcal{B})$. The set of matrices $(\boldsymbol{D}(\mathcal{B}))_{\mathrm{a}}=$ $\left\{\boldsymbol{D}\left(\mathrm{a}^{-1} \mathrm{ba}\right), \mathrm{b} \in \mathcal{B}, \mathrm{a} \in \mathcal{A}\right\}$ is an irrep of $\mathcal{B}$ and is known as conjugate to $\boldsymbol{D}(\mathcal{B})$ (with respect to $\mathcal{A}$ ). It is either equivalent, $(\boldsymbol{D}(\mathcal{B}))_{\mathrm{a}} \sim \boldsymbol{D}(\mathcal{B})$, or non-equivalent to $\boldsymbol{D}(\mathcal{B})$. The set of the non-equivalent irreps $(\boldsymbol{D}(\mathcal{B}))_{\mathrm{a}}$ for all $\mathrm{a} \in \mathcal{A}$ forms the orbit of $\boldsymbol{D}(\mathcal{B})$. The little group $\mathcal{L}(\boldsymbol{D}(\mathcal{B}))$ consists of all those elements a of $\mathcal{A}$ for which $(\boldsymbol{D}(\mathcal{B}))_{\mathrm{a}} \sim \boldsymbol{D}(\mathcal{B})$.

In the special cases of indices 2 and 3 , the little group $\mathcal{L}(\boldsymbol{D}(\mathcal{B}))$ of any irrep $\boldsymbol{D}(\mathcal{B})$ of $\mathcal{B}$ is either the group $\mathcal{A}$ or its normal subgroup $\mathcal{B}$. Two cases are to be distinguished:

(i) The orbit of $\boldsymbol{D}(\mathcal{B})$ has length 2 or $3, \mathcal{L}(\boldsymbol{D}(\mathcal{B}))=\mathcal{B}$.

(ii) The orbit of $\boldsymbol{D}(\mathcal{B})$ has length 1, i.e. $\mathcal{L}(\boldsymbol{D}(\mathcal{B}))=\mathcal{A}$. The irrep $\boldsymbol{D}(\mathcal{B})$ is called self-conjugate.

(a) The induction formulae for orbits of lengths 2 and 3.

For a normal subgroup of index 2 or 3 , one can decompose $\mathcal{A}$ into cosets relative to $\mathcal{B}$, i.e. $\mathcal{A}=\mathcal{B}+\mathrm{q} \mathcal{B}$ for index 2 , and $\mathcal{A}=\mathcal{B}+\mathrm{q} \mathcal{B}+\mathrm{q}^{2} \mathcal{B}$ for index 3 with $\mathrm{q} \in \mathcal{A}$ but $\mathrm{q} \notin \mathcal{B}$.

The orbits of irreps of $\mathcal{B}$ have the form:

index 2:

$$
O(\boldsymbol{D}(\mathcal{B}))=\left\{\boldsymbol{D}(\mathcal{B}),(\boldsymbol{D}(\mathcal{B}))_{\mathrm{q}}\right\}
$$

index 3:

$$
O(\boldsymbol{D}(\mathcal{B}))=\left\{\boldsymbol{D}(\mathcal{B}),(\boldsymbol{D}(\mathcal{B}))_{\mathrm{q}},(\boldsymbol{D}(\mathcal{B}))_{\mathrm{q}^{2}}\right\},
$$

where $(\boldsymbol{D}(\mathcal{B}))_{\mathrm{a}}$ are the non-equivalent conjugate irreps to $\boldsymbol{D}(\mathcal{B})$ with respect to $\mathcal{A}$.

The matrices of the irreps $\boldsymbol{D}^{\text {Ind }}(\mathcal{A})$ of $\mathcal{A}$ for a subgroup of index 2 are given by

$$
\begin{aligned}
& \boldsymbol{D}^{\text {Ind }}(\mathrm{b})=\left(\begin{array}{cc}
\boldsymbol{D}(\mathrm{b}) & \boldsymbol{O} \\
\boldsymbol{O} & (\boldsymbol{D}(\mathrm{b}))_{q}
\end{array}\right) \\
& \boldsymbol{D}^{\text {Ind }}(\mathrm{q})=\left(\begin{array}{cc}
\boldsymbol{O} & \boldsymbol{D}\left(\mathrm{q}^{2}\right) \\
\boldsymbol{I} & \boldsymbol{O}
\end{array}\right) .
\end{aligned}
$$

Similarly, for index 3:

$$
\begin{aligned}
\boldsymbol{D}^{\mathrm{Ind}}(\mathrm{b}) & =\left(\begin{array}{ccc}
\boldsymbol{D}(\mathrm{b}) & \boldsymbol{O} & \boldsymbol{O} \\
\boldsymbol{O} & (\boldsymbol{D}(\mathrm{b}))_{q} & \boldsymbol{O} \\
\boldsymbol{O} & \boldsymbol{O} & (\boldsymbol{D}(\mathrm{b}))_{q^{2}}
\end{array}\right) \\
\boldsymbol{D}^{\mathrm{Ind}}(\mathrm{q}) & =\left(\begin{array}{ccc}
\boldsymbol{O} & \boldsymbol{O} & \boldsymbol{D}\left(\mathrm{q}^{3}\right) \\
\boldsymbol{I} & \boldsymbol{O} & \boldsymbol{O} \\
\boldsymbol{O} & \boldsymbol{I} & \boldsymbol{O}
\end{array}\right) .
\end{aligned}
$$

Each orbit of irreps of $\mathcal{B}$ yields exactly one irrep of $\mathcal{A}$.

(b) Orbits of length 1.

If the length of the orbit of an irrep of $\mathcal{B}$ is 1 , then for the little group $\mathcal{L}(\boldsymbol{D}(\mathcal{B}))=\mathcal{A}$ holds. Every such irrep of $\mathcal{B}$ gives rise to $|\mathcal{A} / \mathcal{B}|$ irreps of $\mathcal{A}$ with the same dimension as $\boldsymbol{D}(\mathcal{B})$ has. The matrices of the irreps $\boldsymbol{D}^{m}(\mathcal{A}), m=1,2$ or $m=1,2,3$, are given as follows. 
For the case of index 2:

$$
\begin{aligned}
& \boldsymbol{D}^{1}(\mathrm{~b})=\boldsymbol{D}^{2}(\mathrm{~b})=\boldsymbol{D}(\mathrm{b}), \quad \mathrm{b} \in \mathcal{B} ; \\
& \boldsymbol{D}^{1}(\mathrm{q})=-\boldsymbol{D}^{2}(\mathrm{q})=\boldsymbol{U},
\end{aligned}
$$

where $\boldsymbol{U}$ is determined by the conditions

$$
\begin{aligned}
\boldsymbol{D}\left(\mathrm{q}^{-1} \mathrm{bq}\right) & =\boldsymbol{U}^{-1} \boldsymbol{D}(\mathrm{b}) \boldsymbol{U}, \quad \mathrm{b} \in \mathcal{B} ; \\
\boldsymbol{U}^{2} & =\boldsymbol{D}\left(\mathrm{q}^{2}\right) .
\end{aligned}
$$

In the case of index 3 :

$$
\begin{aligned}
\boldsymbol{D}^{m}(\mathrm{~b}) & =\boldsymbol{D}(\mathrm{b}), \quad m=1,2,3 ; \\
\boldsymbol{D}^{1}(\mathrm{q}) & =\epsilon \boldsymbol{D}^{2}(\mathrm{q})=\epsilon^{2} \boldsymbol{D}^{3}(\mathrm{q})=\boldsymbol{U}, \\
\text { with } \epsilon & =\exp (2 \pi i / 3),
\end{aligned}
$$

where $\boldsymbol{U}$ is determined by the conditions

$$
\begin{aligned}
\boldsymbol{D}\left(\mathrm{q}^{-1} \mathrm{bq}\right) & =\boldsymbol{U}^{-1} \boldsymbol{D}(\mathrm{b}) \boldsymbol{U}, \quad \mathrm{b} \in \mathcal{B} \quad \text { and } \\
\boldsymbol{U}^{3} & =\boldsymbol{D}\left(\mathrm{q}^{3}\right) .
\end{aligned}
$$

Step 5. Induction procedure for the construction of the irreps

The irreps of a space group $\mathcal{G}$ for a given $\mathbf{k}$ vector are obtained from the allowed irreps $\boldsymbol{D}^{\mathbf{k}, i}$ of the corresponding little group $\mathcal{G}^{\mathbf{k}}$ by induction. Let the elements $\mathrm{q}_{m}=\left(\boldsymbol{W}_{m}, \boldsymbol{w}_{m}\right), m=1, \ldots, s$, be the representatives of the cosets of the decomposition of $\mathcal{G}$ relative to $\mathcal{G}^{\mathbf{k}}$. If $\operatorname{dim}\left(\boldsymbol{D}^{\mathbf{k}, i}\right)=r$ and $s$ is the order of the star of $\mathbf{k}$, then the induced irrep $\boldsymbol{D}^{* \mathbf{k}, i}(\mathcal{G})$ has the dimensions $r \times s$ and its matrices can be written in the form

$$
\boldsymbol{D}^{* \mathbf{k}, i}(\boldsymbol{W}, \boldsymbol{w})_{m p, n q}=\boldsymbol{M}(\boldsymbol{W}, \boldsymbol{w})_{m, n} \boldsymbol{D}^{\mathbf{k}, i}\left(\boldsymbol{W}^{\mathbf{k}}, \boldsymbol{w}^{\mathbf{k}}\right)_{p, q},
$$

where $\left(\boldsymbol{W}^{\mathbf{k}}, \boldsymbol{w}^{\mathbf{k}}\right)=\left(\mathrm{q}_{m}\right)^{-1}(\boldsymbol{W}, \boldsymbol{w}) \mathrm{q}_{n}$ is an element of the little group $\mathcal{G}^{\mathbf{k}}$. The $s$-dimensional matrix $\boldsymbol{M}(\boldsymbol{W}, \boldsymbol{w})$ is the so-called induction matrix. It consists of zeros and ones only, having exactly one ' 1 ' in the $m$ th row and $n$th column, determined by the condition $\left(\mathrm{q}_{m}\right)^{-1}(\boldsymbol{W}, \boldsymbol{w}) \mathrm{q}_{n} \in \mathcal{G}^{\mathbf{k}}$. Correspondingly, the matrices $\boldsymbol{D}^{* \mathbf{k}, i}(\boldsymbol{W}, \boldsymbol{w})$ have block structure with exactly one non-zero $(r \times r)$ block in every column and every row, where the block is the matrix $\boldsymbol{D}^{\mathbf{k}, i}\left(\boldsymbol{W}^{\mathbf{k}}, \boldsymbol{w}^{\mathbf{k}}\right)$ and $\left(\boldsymbol{W}^{\mathbf{k}}, \boldsymbol{w}^{\mathbf{k}}\right)$ is fixed by the above condition. Very often, for a better overview of the irreps of $\mathcal{G}$, their matrices are presented by the non-zero blocks of the induction matrix and the corresponding submatrices of the little-group irreps.

All irreps of the space group $\mathcal{G}$ for a given $\mathbf{k}$ vector are obtained considering all allowed irreps $\boldsymbol{D}^{\mathbf{k}, m}$ of the little group $\mathcal{G}^{\mathbf{k}}$ obtained in Step 4.

\subsubsection{The program REPRES.}

\section{Input information}

(a) Space group data. As input, the program needs the specification of the space group $\mathcal{G}$, which can be defined by its sequential ITA number. Here, as well as in the rest of programs related to space-group representations, the following ITA conventional settings are chosen as default: unique axis $b$ setting for monoclinic groups, hexagonal axes setting for rhombohedral groups, and origin choice 2 for the centrosymmetric groups listed with respect to two origins in ITA. The program REPRES can treat space groups in unconventional settings, once the transformation matrix-
Table 1

Conventional k-vector coefficients $\left(k_{1}, k_{2}, k_{3}\right)$ in terms of primitive (CDML) k-vector coefficients $\left(k_{p 1}, k_{p 2}, k_{p 3}\right)$.

\begin{tabular}{llll}
\hline Lattice types & $k_{1}$ & $k_{2}$ & $k_{3}$ \\
\hline$a P, m P, o P, t P$, & $k_{p 1}$ & $k_{p 2}$ & $k_{p 2}$ \\
$c P, h P, r P$ & & $k_{p 2}-k_{p 3}$ & $k_{p 2}+k_{p 3}$ \\
$m A, o A$ & $k_{p 1}$ & $-k_{p 1}+k_{p 2}$ & $k_{p 3}$ \\
$m C, o C$ & $k_{p 1}+k_{p 2}$ & $k_{p 1}-k_{p 2}+k_{p 3}$ & $k_{p 1}+k_{p 2}-k_{p 3}$ \\
$o F, c F$ & $-k_{p 1}+k_{p 2}+k_{p 3}$ & $k_{p 1}+k_{p 3}$ & $k_{p 1}+k_{p 2}$ \\
$o I, t I, c I$ & $k_{p 2}+k_{p 3}$ & $k_{p 2}-k_{p 3}$ & $k_{p 1}+k_{p 2}+k_{p 3}$ \\
$h R$ (hexagonal) & $k_{p 1}-k_{p 2}$ & &
\end{tabular}

column pair $(\boldsymbol{P}, \boldsymbol{p})$ to the corresponding conventional setting is known. The $3 \times 3$ square matrix $\boldsymbol{P}=\left\|P_{i j}\right\|$ transforms the conventional basis $(\mathbf{a}, \mathbf{b}, \mathbf{c})$ of $\mathcal{G}$ to the non-conventional $\left(\mathbf{a}^{\prime}, \mathbf{b}^{\prime}, \mathbf{c}^{\prime}\right)$ one:

$$
\left(\mathbf{a}^{\prime}, \mathbf{b}^{\prime}, \mathbf{c}^{\prime}\right)=(\mathbf{a}, \mathbf{b}, \mathbf{c}) \boldsymbol{P} .
$$

The column $\boldsymbol{p}=\left(p_{1}, p_{2}, p_{3}\right)$ of coordinates of the nonconventional origin $O^{\prime}$ is referred to the conventional coordinate system of $\mathcal{G}$.

(b) $\mathbf{k}$-vector data. The program accepts $\mathbf{k}$-vector coefficients given with respect to different coordinate systems of the reciprocal space. For its internal calculations, REPRES uses k-vector coefficients $\left(k_{1}, k_{2}, k_{3}\right)$ referred to a basis $\left(\mathbf{a}^{*}, \mathbf{b}^{*}, \mathbf{c}^{*}\right)$ which is dual to the conventional ITA settings of the space groups, $c f$. Step 1 (called conventional k-vector coefficients). The program accepts also k-vector coefficients referred to a primitive basis $\left(\mathbf{p}_{\mathbf{1}}^{*}, \mathbf{p}_{\mathbf{2}}^{*}, \mathbf{p}_{\mathbf{3}}^{*}\right)$ of the reciprocal lattice as given, for example, in CDML tables of space-group irreps. The relations between the conventional and primitive k-vector coefficients are given in Table 1. If a non-conventional setting for the space group is chosen (12), then the corresponding 'non-conventional' k-vector coefficients

$$
\left(k_{1}^{\prime}, k_{2}^{\prime}, k_{3}^{\prime}\right)=\left(k_{1}, k_{2}, k_{3}\right) \boldsymbol{P}
$$

can be given as input data. Note that the program does not accept variables (free parameters) as coefficients of the wavevector. The label of the $\mathbf{k}$ vector asked for by the program is used only for designating the irreps of the little and space groups.

The Brillouin-zones database with wavevector tables for all 230 space groups and figures of the Brillouin zones is available on the Bilbao Crystallographic Server. In the compilation, the symmetry properties of the wavevectors are described by the so-called reciprocal-space groups which are isomorphic to symmorphic space groups (Wintgen, 1941; see also Aroyo \& Wondratschek, 1995). The isomorphism allows the application of crystallographic conventions in the classification of the wavevectors (and henceforth in the irreps of the space groups). For example, the different symmetry types of $\mathbf{k}$ vectors correspond to the different kinds of point orbits (Wyckoff positions) of the symmorphic space groups, the unit cells with asymmetric units given in ITA can serve as Brillouin zones and representation domains etc. The figures and the wavevector data based on the reciprocal-space-group symmetry are compared with the representation domains and 
the $\mathbf{k}$-vector tables of CDML. In the tables, the $\mathbf{k}$-vector data as listed by CDML are compared with the Wyckoff-position description as given in ITA [the so-called adjusted coefficients, cf. Aroyo \& Wondratschek (1995)]. Each k-vector type is specified by its label and parameters. The names of $\mathbf{k}$-vector points, lines and planes of CDML are retained in this listing. New names have been given only to points and lines that are not listed in CDML.

The figures of the Brillouin-zone database available on the Bilbao Crystallographic Server are illustrated by the diagram of the Brillouin zone for the space groups of the arithmetic crystal class $m \overline{3} m F$ displayed in Fig. 1 .

\section{Output information}

The output produced by REPRES contains the following data.

1. Information on the space group $\mathcal{G}$.

(a) Non-translational generators of $\mathcal{G}$ listed as matrixcolumn pairs $(\boldsymbol{W}, \boldsymbol{w})$, i.e. in $(3 \times 4)$ matrix form. The sequence of generators follows that of ITA for the conventional settings of the space groups.

(b) List of translational coset representatives $(\boldsymbol{W}, \boldsymbol{w})$ of $\mathcal{G}$, equation (1), given in $(3 \times 4)$ matrix form. The numbers coincide with the sequential numbers of the general-position coordinate triplets listed in ITA.

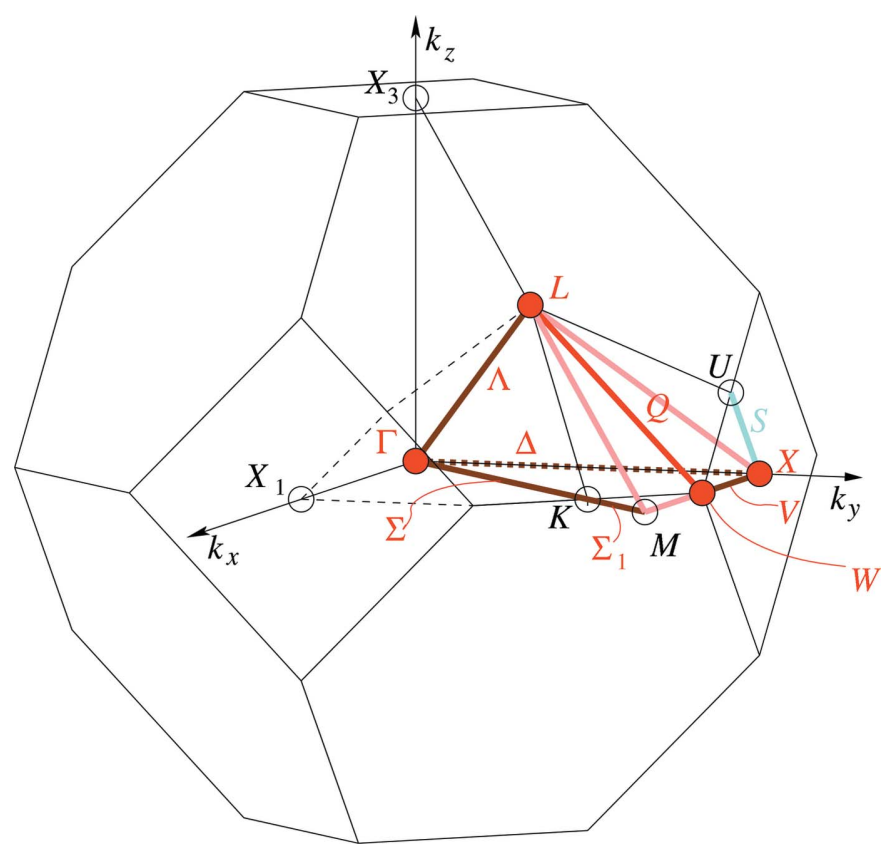

Figure 1

Brillouin-zone diagram for the space groups $F m \overline{3} m, F m \overline{3} c, F d \overline{3} m$ and $F d \overline{3} c$. The asymmetric unit (AU) is the asymmetric tetrahedron $\boldsymbol{\Gamma}, \mathbf{M}, \mathbf{X}, \mathbf{L}$; the representation domain (RD) is the more complicated body with the vertices $\Gamma, \mathbf{K}, \mathbf{W}, \mathbf{X}, \mathbf{U}$ and $\mathbf{L}$. The special points are given in red; the edges of the AU are brown if they are special lines, otherwise they are pink; the red line $\mathbf{L W}$ is special but not an edge of the AU. Owing to the special shape of the RD of CDML, a set of $\mathbf{k}$ vectors of the same type (i.e. that give rise to the same type of irreps) is split into two parts: the special lines $\boldsymbol{\Sigma}$ and $\mathbf{S}$. In the AU description, $\boldsymbol{\Sigma} \cup S$ corresponds to one line $[\Gamma M]$, with $\boldsymbol{\Sigma}_{1} \sim \mathbf{S}$.
2. k-vector data. The program lists the input values of the $\mathbf{k}$-vector coefficients followed by the corresponding conventional coefficients $\left(k_{1}, k_{2}, k_{3}\right)$. The coefficients of the arms of the wavevector star $* \mathbf{k}$ are referred also to the basis that is dual to the conventional (default) setting of the space group.

3. Information on the little group $\mathcal{G}^{\mathbf{k}}$, equation (5).

(a) A set of coset representatives of $\mathcal{G}$ with respect to the little group $\mathcal{G}^{\mathbf{k}}$.

(b) A set of non-translational generators and a set of translational coset representatives of $\mathcal{G}^{\mathbf{k}}$ given as $(3 \times 4)$ matrices.

(c) Little-group irreps presented in a matrix form for the translational coset representatives of $\mathcal{G}^{\mathbf{k}}$ in consecutive order.

4. Full-group representations. The program gives the fullgroup irreducible representations of the non-translational generators of the space group in a block-matrix form: for a given representation and a generator, the program prints out the induction matrix whose non-zero entries, specified by its row and column indices, indicate a matrix block corresponding to a little-group matrix, $c f$. equation (11).

URL of the program: http://www.cryst.ehu.es/rep/ repres.html.

Example: Irreps of $P 4 \mathrm{bm}$ for $\mathbf{k}=\mathbf{X}(0,1 / 2,0)$.

The input data consists in the specification of the space group $P 4 b m$ by its ITA number, No. 100 , and the data for $\mathbf{k}$-vector coefficients, $\mathbf{k}=\mathbf{X}(0,1 / 2,0)$. [In all space groups with primitive lattices, the $\mathbf{k}$-vector coefficients $\left(k_{p 1}, k_{p 2}, k_{p 3}\right)$, referred to a primitive basis of the reciprocal space (CDML), coincide with the conventional $\mathbf{k}$-vector coefficients, $c f$. Table 1.]

The discussion of the output follows the order of the results as they appear in the output file. All space-group elements $(\boldsymbol{W}, \boldsymbol{w})$ are given in a matrix-column form consisting of a $(3 \times 3)$ matrix part $\boldsymbol{W}$ and a $(3 \times 1)$ column part $\boldsymbol{w}$ :

$$
(\boldsymbol{W}, \boldsymbol{w})=\left(\begin{array}{llll}
W_{11} & W_{12} & W_{13} & w_{1} \\
W_{21} & W_{22} & W_{23} & w_{2} \\
W_{31} & W_{32} & W_{33} & w_{3}
\end{array}\right) .
$$

1. The space-group information block contains the following data.

(a) The generators of $P 4 b m$ (with the exception of the generating translations) are listed in the same sequence as they appear in ITA: $(\boldsymbol{1}, \boldsymbol{o}),\left(\boldsymbol{2}_{z}, \boldsymbol{o}\right),\left(\boldsymbol{4}_{z}, \boldsymbol{o}\right),\left(\boldsymbol{m}_{x}, \tau\right)$, with $\tau=(1 / 2,1 / 2,0){ }^{2}$

(b) Decomposition of $\mathrm{P} 4 \mathrm{bm}$ relative to its translation subgroup with coset representatives as given in ITA: $(\boldsymbol{1}, \boldsymbol{o})$, $\left(\boldsymbol{2}_{z}, \boldsymbol{o}\right),\left(\boldsymbol{4}_{z}, \boldsymbol{o}\right),\left(\boldsymbol{4}_{z}^{3}, \boldsymbol{o}\right),\left(\boldsymbol{m}_{x}, \tau\right),\left(\boldsymbol{m}_{y}, \boldsymbol{\tau}\right),\left(\boldsymbol{m}_{x \bar{x}}, \tau\right),\left(\boldsymbol{m}_{x x}, \tau\right)$.

2. The k-vector information block gives the information on the following.

(a) The input k-vector coefficients $\mathbf{X}(0,1 / 2,0)$ followed by the corresponding conventional coefficients.

(b) The k-vector star: $* \mathbf{X}=\{(0,1 / 2,0),(1 / 2,0,0)\}$.

\footnotetext{
${ }^{2}$ To make the description more compact, we use a symbolic notation for the space-group elements.
} 
(c) The little group $\mathcal{G}^{\mathbf{X}}=P 2_{z} b a$ is specified by the coset representatives of its decomposition with respect to the translation subgroup: $(\boldsymbol{1}, \boldsymbol{o}),\left(\boldsymbol{2}_{z}, \boldsymbol{o}\right),\left(\boldsymbol{m}_{y}, \tau\right),\left(\boldsymbol{m}_{x}, \tau\right)$. The little co-group $\overline{\mathcal{G}}^{\mathbf{X}}=\left\{\boldsymbol{1}, \boldsymbol{2}_{z}, \boldsymbol{m}_{x}, \boldsymbol{m}_{y}\right\}$ is isomorphic to the point group $2{ }_{z} \mathrm{~mm}$.

(d) The coset representatives of the decomposition of $P 4 \mathrm{bm}$ relative to $P 2_{z} b a: P 4 b m=P 2_{z} b a+\left(\boldsymbol{4}_{z}, \boldsymbol{o}\right) P 2_{z} b a$.

3. Allowed irreps of $\mathcal{G}^{\mathbf{x}}$ (cf. Step 4 of the REPRES algorithm).

As the little group $\mathcal{G}^{\mathbf{x}}$ is non-symmorphic and the $\mathbf{k}$ vector is on the surface of the Brillouin zone, it is not possible to relate directly the allowed irreps of $P 2_{z} b a$ with the linear irreps of the little co-group $2 \mathrm{~mm}$. The program determines the allowed irreps by constructing the composition series for the little group $P 2_{z} b a$ :

$$
P 2_{z} b a \triangleright P 2_{z} \triangleright \mathcal{T} \text {. }
$$

The allowed irreps of $P 2_{z} b a$ are obtained in two steps.

(a) Construction of the allowed irreps of $P 2_{z}$ starting from those of the translational subgroup $\mathcal{T}$ :

\begin{tabular}{l||cc|c}
$P 2_{z}$ & $(\boldsymbol{1}, \boldsymbol{o})$ & $(\mathbf{2}, \boldsymbol{o})$ & $(\boldsymbol{1}, \boldsymbol{t})$ \\
\hline $\boldsymbol{D}_{P 2_{z}}^{\mathbf{X}, 1}$ & 1 & 1 & $\exp -\left(i \pi n_{2}\right)$ \\
$\boldsymbol{D}_{P 2_{z}}^{\mathbf{X}, 2}$ & 1 & -1 & $\exp -\left(i \pi n_{2}\right)$
\end{tabular}

Here, $\boldsymbol{t}$ is the column of coefficients $\left(n_{1}, n_{2}, n_{3}\right)$.

(b) From the conjugation of the elements of $P 2_{z}$ under $\left(\boldsymbol{m}_{x}, \tau\right)$, it follows that the two allowed irreps of $P 2_{z}$ form an orbit of conjugate irreps, i.e. there is just one allowed twodimensional irrep of $P 2_{z} b a$, equation (7).

\begin{tabular}{c||ccc}
$P 2_{z} b a$ & $(\mathbf{2}, \boldsymbol{o})$ & $\left(\boldsymbol{m}_{x}, \tau\right)$ & $\left(\boldsymbol{m}_{y}, \tau\right)$ \\
\hline $\boldsymbol{D}^{\mathbf{X}, 1}$ & $\left(\begin{array}{cc}1 & 0 \\
0 & -1\end{array}\right)$ & $\left(\begin{array}{cc}0 & -1 \\
1 & 0\end{array}\right)$ & $\left(\begin{array}{ll}0 & 1 \\
1 & 0\end{array}\right)$.
\end{tabular}

The program lists the corresponding irrep matrices following the consecutive order of the translational coset representatives of the little group. The (complex) matrix elements are specified by their moduli and phase angles in degrees [ $\left.{ }^{\circ}\right]$. For example, the matrix of the element $\left(\boldsymbol{m}_{x}, \tau\right)$, listed under No. 4 , has the form:

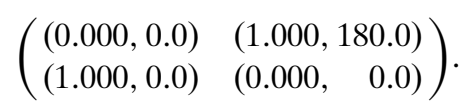

4. Full-group irreps (cf. Step 5 of the REPRES algorithm). The matrices of the full-group irreps for the non-translation generators are presented in a block-matrix form. The program lists separately the induction matrix $\boldsymbol{M}(\boldsymbol{W}, \boldsymbol{w})$ and the corresponding blocks of the little-group representation matrices [equation (11)] specified by the row-column indices of the non-zero entries of $\boldsymbol{M}(\boldsymbol{W}, \boldsymbol{w})$. For example, the matrix of the full-group irrep for the generator $\left(\boldsymbol{4}_{z}, \boldsymbol{o}\right)$ of $\mathrm{P} 4 \mathrm{bm}$ (No. 3 in the list of generators),

$$
\boldsymbol{D}^{* \mathbf{X}, 1}\left(\boldsymbol{4}_{z}, \boldsymbol{o}\right)=\left(\begin{array}{cc|cc}
0 & 0 & 1 & 0 \\
0 & 0 & 0 & 1 \\
\hline 1 & 0 & 0 & 0 \\
0 & 1 & 0 & 0
\end{array}\right),
$$

is presented as a $(2 \times 2)$ induction matrix

$$
\boldsymbol{M}^{* \mathbf{X}, 1}\left(\boldsymbol{4}_{z}, \boldsymbol{o}\right)=\left(\begin{array}{cc}
0 & 1 \\
1 & 0
\end{array}\right)
$$

with the following $(2 \times 2)$ blocks:

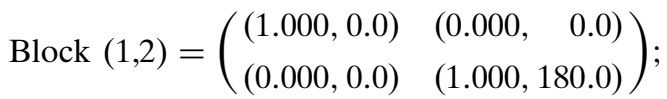

$$
\begin{aligned}
& \text { Block }(2,1)=\left(\begin{array}{cc}
(1.000,0.0) & (0.000,0.0) \\
(0.000,0.0) & (1.000,0.0)
\end{array}\right) \text {. }
\end{aligned}
$$

\subsection{Point-group representations (POINT)}

2.2.1. The problem. The information about the 32 (nonmagnetic) crystallographic point groups plays a fundamental role in many applications of crystallography. Examples of such applications range from the external shape and physical properties of macroscopic crystals to the local site symmetry in crystal structures. In the literature, there exists a lot of information about crystallographic point groups and their representations. Some complete tables are given in Koster et al. (1963), Bradley \& Cracknell (1972), Altmann \& Herzig (1994) (and references therein). In our case, a selection of these data has been recalculated and is now available online via the Bilbao Crystallographic Server. The point-group databases are part of the core shell of the server. They provide essential information for a point-group analysis in applications related to crystallographic, solid-state or phase-transition problems.

2.2.2. The method. The information about the irreps of the 32 point groups can be obtained from the program REPRES for the particular case of $* \mathbf{k}=\boldsymbol{\Gamma}$. All generated point-group data have been stored as an XML database of the server. A physical property can be represented by a tensor that transforms, in general, according to a reducible representation. Any reducible representation can be decomposed into irreducible constituents applying the so-called reduction ('magic') formula:

$$
\Gamma \sim \bigoplus_{i} n_{i} \Gamma_{i}, \quad \text { where } \quad n_{i}=\frac{1}{|\overline{\mathcal{G}}|} \sum_{\mathrm{g}} \chi(\mathrm{g}) \chi_{i}^{*}(\mathrm{~g}) .
$$

In the last expression, $\Gamma$ represents a reducible representation expressed in terms of its irreps $\Gamma_{i}$. The multiplicity of the irrep is given by $n_{i},|\overline{\mathcal{G}}|$ is the order of the point group, i.e. the number of symmetry operations of the point group, and $\chi(\mathrm{g})$ and $\chi_{i}(\mathrm{~g})$ are the corresponding characters of the reducible representation $\Gamma$ and the irrep $\Gamma_{i}$.

2.2.3. The program POINT. The program POINT displays a set of several tables for each of the 32 crystallographic point groups that are specified by their international (HermannMauguin) and Schoenflies symbols. 
1. Character table. The character table provides the characters of the ordinary (vector) irreps of a chosen point group. The irreps are labeled in the notation of Mulliken (1933) and by the $\Gamma$ labels given by Koster et al. (1963). The matrices of the degenerate irreps as calculated by REPRES are also accessible. The number of point-group elements in a conjugacy class is indicated by the listed multiplicity. In addition, the transformation properties of the Cartesian tensors of ranks 1 (vectors and axial vectors) and 2 are displayed. (The tensor of rank 0 always belongs to the totally symmetric irrep and is not listed explicitly). Cartesian tensors that span two- or threedimensional irreps are joined by brackets.

2. Subgroup table. The point-group types of the subgroups of a point group are listed with the corresponding indices in the initial point group.

3. Irrep multiplication table. The table shows the decomposition into irreducible constituents of the Kronecker (direct) product of any pair of point-group irreps.

4. Tensor representations. The table lists the decompositions into irreducible constituents of representations related to some important tensors (and their powers), such as the vector $V$ (polar) or the pseudovector $A$ (axial), their symmetrized $\left[V^{2}\right]$ or antisymmetrized squares etc.

5. Selection rules for fundamental transitions. The table displays the selection rules for infrared and Raman electronic transitions. The data in the first row of each table (specified by the trivial irrep label) correspond to the usual infrared and Raman selection rules.

6. Subduction from the rotation group irreps. Given a (vector) representation of the rotation group of dimension $2 l+1, l=0, \ldots, 9$, the table lists the point-group irreps that appear in its subduction to the chosen point group.

URL of the program: http://www.cryst.ehu.es/rep/ point.html.

\section{Correlations between the representations of group- subgroup-related space groups}

\subsection{The problem}

For different physical applications, it is important to know the compatibility relations (known also as correlations) between the representations of group-subgroup-related crystallographic groups $\mathcal{H}<\mathcal{G}$. For their calculation, it is sufficient to consider the irreps of $\mathcal{G}$. The problem can be formulated as follows: given an irrep $\boldsymbol{D}_{\mathcal{G}}$ of $\mathcal{G}$, how does the subduced representation $\boldsymbol{D}_{\mathcal{G}}^{s}=\boldsymbol{D}_{\mathcal{G}} \downarrow \mathcal{H}$ decompose into irreps of $\boldsymbol{D}_{\mathcal{H}}$ of $\mathcal{H}$ ? This subduced representation $\boldsymbol{D}_{\mathcal{G}}^{s}$ is in general a reducible one and can be transformed into a direct sum of irreps $\boldsymbol{D}_{\mathcal{H}}$ of $\mathcal{H}$ by a suitable unitary transformation $\boldsymbol{S}$ :

$$
\boldsymbol{D}_{\mathcal{G}}^{s} \boldsymbol{S}=\boldsymbol{S}\left[\oplus \boldsymbol{E}(s \mid i) \otimes \boldsymbol{D}_{\mathcal{H}}^{i}\right],
$$

where $\boldsymbol{E}(s \mid i)$ is the unit matrix of dimension $(s \mid i)$ and $(s \mid i)$ is the number of times the irrep $\boldsymbol{D}_{\mathcal{H}}^{i}$ is contained in the $\boldsymbol{D}_{\mathcal{G}}^{s}$ (the so-called subduction coefficients). The matrix $\boldsymbol{S}$ is known as the subduction matrix.
Subduction coefficients and subduction matrices occur in various physical problems. For example, in crystal-field theory the subduction coefficients indicate how the energy level of a free atom splits under the influence of the crystal field while the corresponding subduction matrices are used to transform the wavefunctions of the free atom to a symmetry-adapted basis which simplifies the eigenvalue problem of the full Hamiltonian. The subduction coefficients and subduction matrices are also necessary for the investigation of phase transitions in solid-state materials, energy-band calculations, morphic effects in Raman and infrared spectra (see e.g. Birman, 1974) etc.

The problem of subduction coefficients and subduction matrices for crystallographic point groups is completely solved. For example, data on subduction coefficients for the irreps of point groups have been tabulated long ago (Koster et al., 1963). However, similar results on subduction quantities for crystallographic space groups are only partially known. The difficulties in their tabulation are related to the great number and variety of space-group representations and possible group-subgroup relations between space groups.

The software package CORREL computes the correlations between representations of a crystallographic space group $\mathcal{G}$ and those of its subgroups $\mathcal{H}$, including the multiplicities of $\boldsymbol{D}_{\mathcal{H}}^{i}$ in $\boldsymbol{D}_{\mathcal{G}} \downarrow \mathcal{H}$. In addition, the program determines the corresponding subduction matrices $\boldsymbol{S}$, which relate the bases of the irreducible constituents $\boldsymbol{D}_{\mathcal{H}}^{i}$ to those of the subduced representation $\boldsymbol{D}_{\mathcal{G}} \downarrow \mathcal{H}$, equation (14).

\subsection{The method}

The space-group formulation of the subduction problem is straightforward. The subduced representation $\boldsymbol{D}^{* \mathbf{k}_{g}, i} \downarrow \mathcal{H}$ is in general a reducible one and can be transformed into a direct sum of irreps $\boldsymbol{D}^{\boldsymbol{k}_{\mathcal{H}}, j}$ of $\mathcal{H}$ by a suitable transformation $\boldsymbol{S}$ :

$$
\left(\boldsymbol{D}^{* \mathbf{k}_{\mathcal{G}}, i} \downarrow \mathcal{H}\right) \boldsymbol{S}=\boldsymbol{S}\left[\oplus \boldsymbol{E}\left(* \mathbf{k}_{\mathcal{G}}, i \mid * \mathbf{k}_{\mathcal{H}}, j\right) \otimes \boldsymbol{D}^{* \mathbf{k}_{\mathcal{H}}, j}\right] .
$$

The direct sum is over all stars $* \mathbf{k}_{\mathcal{H}}$ of $\mathcal{H}$ and over all allowable irreps $\boldsymbol{D}^{* \mathbf{k}_{\mathcal{H}}, j}$ that may arise for a given $* \mathbf{k}_{\mathcal{H}}$. The subduction coefficients $\left(* \mathbf{k}_{\mathcal{G}}, i \mid * \mathbf{k}_{\mathcal{H}}, j\right)$ are integers and denote the multiplicity of irreps $\boldsymbol{D}^{* \mathbf{k}_{\mathcal{H}}, j}$ in the subduced representation. The rows of the subduction matrix $\boldsymbol{S}$ are labeled by the row indices of $\boldsymbol{D}^{* \mathbf{k}_{g}, i}$. The columns of the subduction matrix are specified by a triple of indices indicating the irrep $\boldsymbol{D}^{* \mathbf{k}_{\mathcal{H}}, j}$, its multiplicity and a row index. The coefficients of a given column of $\boldsymbol{S}$ determine the linear combination of the basis functions of $\boldsymbol{D}^{* \mathbf{k}_{g}, i}$ that transforms as the corresponding row of $\boldsymbol{D}^{* \mathbf{k}_{\mathcal{H}}, j}$.

We are interested in the calculation of the subduction coefficients and the subduction matrix $\boldsymbol{S}$.

In order to determine the subduction coefficients $\left(* \mathbf{k}_{\mathcal{G}}, i \mid * \mathbf{k}_{\mathcal{H}}, j\right)$, one can proceed by rewriting the defining equation (15) using the character systems $\chi^{* \mathbf{k}_{G}, i}$ of $\boldsymbol{D}^{* \mathbf{k}_{\mathcal{G}}, i}$ and $\chi^{* \mathbf{k}_{\mathcal{H}}, j}$ of $\boldsymbol{D}^{* \mathbf{k}_{\mathcal{H}}, j}$ :

$$
\chi^{* \mathbf{k}_{\mathcal{G}}, i}(\mathrm{~h})=\sum\left(* \mathbf{k}_{\mathcal{G}}, i \mid * \mathbf{k}_{\mathcal{H}}, j\right) \chi^{* \mathbf{k}_{\mathcal{H}}, j}(\mathrm{~h}), \quad \mathrm{h} \in \mathcal{H} .
$$


The application of the orthogonality properties of the characters of the irreps $\boldsymbol{D}^{* \mathbf{k}_{\mathcal{H}}, j}$ to equation (16) results in an expression for the subduction coefficients that is difficult to use directly: it would involve a sum over all elements of the subgroup. An alternative to the conventional routine for determination of $\left(* \mathbf{k}_{\mathcal{G}}, i \mid * \mathbf{k}_{\mathcal{H}}, j\right)$ follows directly from their definition. Although the sum in equation (15) is over all stars $* \mathbf{k}_{\mathcal{H}}$, in fact only representations from a small number of stars are contained in the subduced representation. The determination of the splitting of the star $* \mathbf{k}_{\mathcal{G}}$ of $\mathcal{G}$ into stars $* \mathbf{k}_{\mathcal{H}}$ of $\mathcal{H}$ is the first step in the procedure for the determination of the subduction coefficients. Owing to the decomposability of the subduced representations into irreducible constituents (15), the star $* \mathbf{k}_{\mathcal{G}}$ is decomposed into entire stars $* \mathbf{k}_{\mathcal{H}}$. One can formally introduce $* \mathbf{k}$ subduction coefficients $\left(* \mathbf{k}_{\mathcal{G}} \mid * \mathbf{k}_{\mathcal{H}}\right)$ to describe the splitting of the star $* \mathbf{k}_{\mathcal{G}}$ :

$$
* \mathbf{k}_{\mathcal{G}}=\sum_{* \mathbf{k}_{\mathcal{H}}}\left(* \mathbf{k}_{\mathcal{G}} \mid * \mathbf{k}_{\mathcal{H}}\right) * \mathbf{k}_{\mathcal{H}},
$$

where the sum is over the stars $* \mathbf{k}_{\mathcal{H}}$ that occur in $* \mathbf{k}_{\mathcal{G}}$. The coefficients $\left(* \mathbf{k}_{\mathcal{G}} \mid * \mathbf{k}_{\mathcal{H}}\right)$ are integers and they relate in an obvious way the number of arms $s_{\mathbf{k}_{\mathcal{G}}}$ and $s_{\mathbf{k}_{\mathcal{H}}}$ of the stars $* \mathbf{k}_{\mathcal{G}}$ and $* \mathbf{k}_{\mathcal{H}}$ :

$$
s_{\mathbf{k}_{\mathcal{G}}}=\sum\left(* \mathbf{k}_{\mathcal{G}} \mid * \mathbf{k}_{\mathcal{H}}\right) s_{\mathbf{k}_{\mathcal{H}}} .
$$

The $* \mathbf{k}$ subduction coefficients are determined by direct inspection: starting from an arbitrary arm of $* \mathbf{k}_{\mathcal{G}}$, one determines all those arms $\mathbf{k}_{\mathcal{G}}$ that belong to the corresponding star $* \mathbf{k}_{\mathcal{H}}$. If any vectors $\mathbf{k}_{\mathcal{G}}$ are left, one continues with the procedure until all $* \mathbf{k}_{\mathcal{G}}$ arms are distributed into $* \mathbf{k}_{\mathcal{H}}$ stars.

The determination of the $* \mathbf{k}$ subduction coefficients reduces the sum in equation (16) and in this way simplifies the calculation of the subduction coefficients. Consider a star $* \mathbf{k}_{\mathcal{H}}$ with $\left(* \mathbf{k}_{\mathcal{G}} \mid * \mathbf{k}_{\mathcal{H}}\right) \neq 0$ whose $n_{\mathbf{k}_{\mathcal{H}}}$ irreps are of dimensions $d^{* \mathbf{k}_{\mathcal{H}}, j}=$ $\operatorname{dim}\left(\boldsymbol{D}^{* \mathbf{k}_{\mathcal{H}}, j}\right)$. A set of linear algebraic equations with the subduction coefficients as unknowns is obtained taking equations (16) for different elements of $\mathcal{H}$. Their number equals $\sum n_{\mathbf{k}_{\mathcal{H}}}$, where the sum is over the number of distinct $* \mathbf{k}_{\mathcal{H}}$ stars that occur in $* \mathbf{k}_{\mathcal{G}}$. The subduction coefficients for given $* \mathbf{k}_{\mathcal{H}}$ are further restricted by the following relation:

$$
\sum_{j}\left(* \mathbf{k}_{\mathcal{G}}, i \mid * \mathbf{k}_{\mathcal{H}}, j\right) d^{* \mathbf{k}_{\mathcal{H}}, j}=r_{\mathbf{k}_{\mathcal{G}}}\left(* \mathbf{k}_{\mathcal{G}} \mid * \mathbf{k}_{\mathcal{H}}\right) s_{\mathbf{k}_{\mathcal{H}}} .
$$

Here $r_{\mathbf{k}_{g}}$ is the dimension of the little-group irrep $\boldsymbol{D}^{\mathbf{k}_{g}, i}$ and the sum is over all irreps $\boldsymbol{D}^{* \mathbf{k}_{\mathcal{H}}, j}$ of $* \mathbf{k}_{\mathcal{H}}$. This condition becomes obvious if one notes that the two sides of the equation express the total dimension of the irreps of $* \mathbf{k}_{\mathcal{H}}$ that occur as irreducible constituents in the decomposition of the subduced representation.

Once the subduction coefficients are determined, it is possible to construct the block-diagonal representation $\oplus \boldsymbol{E}\left(* \mathbf{k}_{\mathcal{G}}, i \mid * \mathbf{k}_{\mathcal{H}}, j\right) \otimes \boldsymbol{D}^{* \mathbf{k}_{\mathcal{H}}, j}$, equivalent to the subduced representation $\left(\boldsymbol{D}^{* \mathbf{k}_{g}, i} \downarrow \mathcal{H}\right)$. The set of matrix equations (15) for the elements of $\mathcal{H}$ forms a system of linear equations with the elements of the subduction matrix $\boldsymbol{S}$ as unknowns. For the explicit calculation of the elements of the subduction matrix it is convenient to split and rewrite equation (15) for each $\boldsymbol{D}^{* \mathbf{k}_{\mathcal{H}}, j}$ separately:

$$
\left(\boldsymbol{D}^{* \mathbf{k}_{\mathcal{G}}, i} \downarrow \mathcal{H}\right) \boldsymbol{S}^{* \mathbf{k}_{\mathcal{H}}, j}=\boldsymbol{S}^{* \mathbf{k}_{\mathcal{H}}, j} \boldsymbol{D}^{* \mathbf{k}_{\mathcal{H}}, j} .
$$

Here the rectangular matrices $\boldsymbol{S}^{* \mathbf{k}_{\mathcal{H}}, j}$ consist of $d^{* \mathbf{k}_{\mathcal{H}}, j}$ columns of $\boldsymbol{S}$ and correspond to the elements of the subduction matrix associated with the irrep $\boldsymbol{D}^{* \mathbf{k}_{\mathcal{H}}, j}$. The number of independent solutions of (20) equals the multiplicity of $\boldsymbol{D}^{* \mathbf{k}_{\mathcal{H}}, j}$ in the subduced representation. One should note that: (i) we are interested only in the nonsingular solutions for the subduction matrix, and (ii) the freedom in the determination of $\boldsymbol{S}$ follows from the Schur lemma for reducible representations: the subduction matrix $\boldsymbol{S}$ is determined up to a matrix belonging to the commuting algebra of the representation $\oplus \boldsymbol{E}\left(* \mathbf{k}_{\mathcal{G}}, i \mid * \mathbf{k}_{\mathcal{H}}, j\right) \otimes \boldsymbol{D}^{* \mathbf{k}_{\mathcal{H}}, j}$.

\subsection{The program CORREL}

As input data, the program requires the groups $\mathcal{G}$ and $\mathcal{H}$ specified by their ITA numbers, and a transformation matrixcolumn pair $(\boldsymbol{P}, \boldsymbol{p})$ relating the conventional (default) bases of the group and the subgroup, equation (12). The k-vector coefficients could be referred to the primitive bases of reciprocal space (of the supergroup) as found in CDML. Another possibility for the cases of centered lattices is to refer the $\mathbf{k}$ vector to the centered dual basis or to specify it by the adjusted coefficients ( $c f$. §2.1.3).

First, the $* \mathbf{k}$ subduction coefficients $\left(* \mathbf{k}_{\mathcal{G}} \mid * \mathbf{k}_{\mathcal{H}}\right)$ and the corresponding subduction coefficients $\left(* \mathbf{k}_{\mathcal{G}}, i \mid * \mathbf{k}_{\mathcal{H}}, j\right)$ are computed. Although the procedure for the determination of * $\mathbf{k}$ subduction coefficients is straightforward, it is worth pointing out that attention should be paid in the cases when the reciprocal lattices of the group and the subgroup are different (e.g. when $\mathcal{H}$ is a klassengleiche subgroup of $\mathcal{G}$ ). The splitting of the star $* \mathbf{k}_{\mathcal{G}}$ into the stars of the subgroup $* \mathbf{k}_{\mathcal{H}}$ is carried out with respect to the subgroup bases. Next, the subduction coefficients are computed by solving the system of linear equations (16). The irreps of $\mathcal{G}$ and $\mathcal{H}$ and their characters necessary for the construction of the system (16) are calculated by REPRES. Trying with different elements of $\mathcal{H}$, the program searches for $\sum n_{\mathbf{k}_{\mathcal{H}}}$ (the sum is over all $* \mathbf{k}_{\mathcal{H}}$ stars obtained from the splitting of $* \mathbf{k}_{\mathcal{G}}$ ) linear independent equations of the type (16). A procedure GAUSS based on the Gauss elimination method solves the system of linear equations and determines the subduction coefficients $\left(* \mathbf{k}_{\mathcal{G}}, i \mid * \mathbf{k}_{\mathcal{H}}, j\right)$.

For the calculation of the subduction matrices, the program constructs the matrices of the direct-sum representation [cf. the right-hand side of equation (20)] using the calculated subduction coefficients and the corresponding matrices of irreps calculated by REPRES. The elements of the subduction matrix $\boldsymbol{S}$ are obtained as solutions of the system of linear equations (20). A modification of the Gauss elimination procedure that takes into account the sparse character of the involved matrices is applied for the purpose.

The output consists of five main subblocks. The listed data start with information on the studied group-subgroup pair of 
space groups $\mathcal{G}>\mathcal{H}$ including their space-group numbers and lattice types, lists of generators and translational coset representatives $(\boldsymbol{W}, \boldsymbol{w})$ given in $(3 \times 4)$ matrix form, and the transformation matrix-column pair $(\boldsymbol{P}, \boldsymbol{p})$. The $* \mathbf{k}_{\mathcal{G}}$ vector is specified by its input coefficients and those referred to the dual basis of the default setting of $\mathcal{G}$, followed by a list of the arms of $* \mathbf{k}_{\mathcal{G}}$.

Under the heading 'INFORMATION FOR THE SPLITTING' follows a block containing information on the splitting of the star $* \mathbf{k}_{\mathcal{G}}$ into wavevector stars $* \mathbf{k}_{\mathcal{H}}$ of the subgroup, $c f$. equation (17).

Next follow two blocks of the same type for the group and the subgroup (with the headings 'INFORMATION FOR THE SUPERGROUP' and 'INFORMATION FOR THE SUBGROUP'), containing information on the relevant little groups, the allowed little-group irreps, the chosen coset representatives of the decomposition of the group with respect to the little group and the full space-group irreps (optional).

The last block of the output, entitled 'SUBDUCTION PROBLEM', contains the subduction coefficients and the corresponding subduction matrices for each irrep of the supergroup for the considered $* \mathbf{k}_{\mathcal{G}}$ vector.

URL of the program: http://www.cryst.ehu.es/rep/ correl.html.

Example. The program CORREL is illustrated by the calculation of the subduction coefficients and matrices for the irrep $\boldsymbol{D}^{* \mathbf{X}, 1}$ of $P 4 b m[\mathbf{k}=\mathbf{X}(0,1 / 2,0)]$ in its subduction to the subgroup $P 2$ of index 8.

The input data includes the following.

1. The specification of the group-subgroup pair by the corresponding ITA numbers of the involved space groups and the transformation matrix $(\boldsymbol{P}, \boldsymbol{p})$ which relates the conventional bases of $\mathcal{G}$ and $\mathcal{H}$. The transformation matrices for a given group-subgroup pair and their index can be obtained from the program SUBGROUPGRAPH available on the Bilbao Crystallographic Server. There are seven subgroups $P 2$ (No. 3) of P4bm (No. 100) of index 8 distributed into four different classes of conjugate subgroups. Here, we consider the symmetry break $P 4 b m>P 2$ with

$$
(\boldsymbol{P}, \boldsymbol{p})=\left(\begin{array}{ccc|c}
0 & 0 & 1 & 0 \\
2 & 0 & 0 & \frac{1}{2} \\
0 & 1 & 0 & 0
\end{array}\right)
$$

2. The wavevector $\mathbf{X}$ is specified by its primitive coefficients $\left(0, \frac{1}{2}, 0\right)$.

In the first block of the output file, the program lists the generators, general positions of $P 4 b m$ and $P 2$ as $(3 \times 4)$ matrix-column pairs. The transformation matrix $(\boldsymbol{P}, \boldsymbol{p})$ is printed in the same form. The coefficients of its translation part are printed as multiples of $\frac{1}{24}$ (e.g. $\frac{1}{2}$ is listed as 12). The two arms of $* \mathbf{X}$ are given by their conventional coefficients $* \mathbf{X}=\left\{\left(0, \frac{1}{2}, 0\right),\left(\frac{1}{2}, 0,0\right)\right\}$.

The splitting of the $* \mathbf{X}$ in the subgroup wavevector stars $* \mathbf{S 1}$ and $* \mathbf{S 2}$, equation (17), is indicated in the next block:

$$
* \mathbf{X}=1 \_* \mathbf{S 1} \oplus 1 \_* \mathbf{S 2},
$$

where each $* \mathbf{k}_{\mathcal{H}}$ is preceded by its multiplicity in $* \mathbf{k}_{\mathcal{G}}$. In our case, $(* \mathbf{X} \mid * \mathbf{S 1})=(* \mathbf{X} \mid * \mathbf{S 2})=1$. The star $* \mathbf{S 1}=(1,0,0)$ is equivalent to the $\boldsymbol{\Gamma}$ wave vector of $P 2$. The star $* \mathbf{S} \mathbf{2}=\left(0,0, \frac{1}{2}\right)$ is also a special one and corresponds to the point $\mathbf{Z}$ in CDML notation ( $c f$. the Brillouin-zone database available on the server).

The next block includes the data on the little group and (optionally) the full-group irreps of $\mathrm{P} 4 \mathrm{bm}$ for the $\mathbf{X}$ wavevector. The little- and full-group irreps of $* \mathbf{S 1}$ and $* \mathbf{S 2}$ of the subgroup $P 2$, relevant for the subduction, are given in the block 'Information about the subgroup'. The presentation of the data follows the form and the sequence already discussed and illustrated in $\$ 2.1 .3$. There is one four-dimensional full group irrep $(* \mathbf{X})(1) \quad(c f$. the example of the program $R E P R E S$ ). For each subgroup $\operatorname{star} * \mathbf{S 1}$ and $* \mathbf{S} 2$ there exist two irreps labeled as $(* \mathbf{S i})(j), i, j=1,2$, i.e. there are altogether four one-dimensional irreps of $P 2$ that can take part in the decomposition of $(* \mathbf{X})(1)$ [cf. equation (15)]. The irrep $(* \mathbf{S 1})(1)$ corresponds to the identity irrep of $P 2$.

The last block of the output file includes the subduction coefficients $\left(* \mathbf{k}_{\mathcal{G}}, i \mid * \mathbf{k}_{\mathcal{H}}, j\right)$ and the subduction matrices $\boldsymbol{S}$. The subduced irrep $(* \mathbf{X})(1) \downarrow P 2$ splits into the four irreps of the subgroup, each appearing with multiplicity 1 :

$$
\begin{aligned}
(* \mathbf{X})(1) & \downarrow P 2 \\
\sim & \sim(* \mathbf{S 1})(1) \oplus(* \mathbf{S 1})(2) \oplus(* \mathbf{S} \mathbf{2})(1) \oplus(* \mathbf{S} \mathbf{2})(2) .
\end{aligned}
$$

The subduction matrix $\boldsymbol{S}$ is shown in two parts: a matrix with numerical values gives one possible (non-zero) solution for the subduction matrix. The irrep indices of its columns follow the order of the irreps in the direct-sum decomposition of $\boldsymbol{D}^{* \mathbf{k}_{g}, i} \downarrow \mathcal{H}$ [equation (21)]. The block-diagonal (unitary) matrix with complex parameters expressed by letters is related to the uniqueness of $\boldsymbol{S}$. The freedom in the determination of the rectangular matrices $\boldsymbol{S}^{* \mathbf{k}_{\mathcal{H}}, j}$ (20) is given by the corresponding subblocks. Their dimensions equal the multiplicities of $\boldsymbol{D}^{* \mathbf{k}_{\mathcal{H}}, j}$ in $\boldsymbol{D}^{* \mathbf{k}_{\mathcal{G}}, i} \downarrow \mathcal{H}$. In our example, all irreps of the subgroup in equation (21) are of dimension 1 and the corresponding rectangular matrices are reduced to single columns. The freedom in their determination is given by single complex numbers (or phase factors if the normalization condition is imposed) as the multiplicities of the irreps $(* \mathbf{S i})(j)$ in $(* \mathbf{X})(1)$ equal 1.

\section{Direct product of representations}

\subsection{The problem}

There are a number of physical applications of the representation theory of space groups that are based on the analysis of the Kronecker product of space-group representations. A well known example is related to the determination of selection rules for various physical processes in crystalline materials as infrared absorption, Raman scattering, neutron scattering, electron scattering etc. [see e.g. Birman \& Berenson (1974) and Cracknell (1974)]. The essential step of the selection-rules derivations consists in the reduction of Kronecker products of space-group representations into irreducible 
constituents. Consider the irreps $\boldsymbol{D}^{* \mathbf{k}, m}$ and $\boldsymbol{D}^{* \mathbf{k}^{\prime}, m^{\prime}}$ of the space group $\mathcal{G}$ spanned by the sets of basis functions $\left\{\phi_{1}^{k_{1}, m}, \ldots, \phi_{r_{s}^{\prime}, m}^{k_{s}, m}\right\}$ and $\left\{\phi_{1}^{k_{1}^{\prime}, m^{\prime}}, \ldots, \phi_{r^{\prime}}^{k_{s^{\prime}}^{\prime}, m^{\prime}}\right\}$. The set of all bilinears $\left\{\phi_{1}^{k_{1}, m} \phi_{1}^{k_{1}^{\prime \prime m}, m^{\prime}}, \ldots, \phi_{r_{m}}^{k_{s}, m} \phi_{r^{\prime}}^{k_{s^{\prime}}, m^{\prime}}\right\}$ form a carrier space of the so-called Kronecker (direct or tensor) product representation $\boldsymbol{D}^{* \mathbf{k}, m} \otimes \boldsymbol{D}^{* \mathbf{k}^{\prime}, m^{\prime}}$ of $\mathcal{G}$. It is in general a reducible representation with $\operatorname{dim}\left(\boldsymbol{D}^{* \mathbf{k}, m} \otimes \boldsymbol{D}^{* \mathbf{k}^{\prime}, m^{\prime}}\right)=\left(s \times r_{m}\right)\left(s^{\prime} \times r_{m^{\prime}}^{\prime}\right) .^{3}$ In order to decompose the direct-product representation into irreducible constituents, one first determines the so-called reduction coefficients of the Clebsch-Gordan series $\left(* \mathbf{k} m, * \mathbf{k}^{\prime} m^{\prime} \mid * \mathbf{k}^{\prime \prime} m^{\prime \prime}\right)$ (in the following referred to as reduction coefficients for short). The reduction coefficients are integers that indicate the irreps $\boldsymbol{D}^{* \mathbf{k}^{\prime \prime}, m^{\prime \prime}}$ that appear in the decomposition of the Kronecker product:

$$
\begin{aligned}
\boldsymbol{D}^{* \mathbf{k}, m} & \otimes \boldsymbol{D}^{* \mathbf{k}^{\prime}, m^{\prime}} \\
& \sim \bigoplus_{* \mathbf{k}^{\prime \prime}, m^{\prime \prime}}\left[\boldsymbol{E}\left(* \mathbf{k} m, * \mathbf{k}^{\prime} m^{\prime} \mid * \mathbf{k}^{\prime \prime} m^{\prime \prime}\right) \otimes \boldsymbol{D}^{* \mathbf{k}^{\prime \prime}, m^{\prime \prime}}\right] .
\end{aligned}
$$

In order to complete the decomposition, one further determines the Clebsch-Gordan coefficients. They define, the correct linear combination of bilinear products $\left(\phi_{\mu}^{k_{\alpha}, m} \phi_{\mu^{\sigma^{\prime}}}^{k_{\sigma^{\prime}}^{\prime}, m^{\prime}}\right)$ which transform according to the irreps $\boldsymbol{D}^{* \mathbf{k}^{\prime \prime}, m^{\prime \prime}}$. The ClebschGordan coefficients can be suitably assembled into a matrix $\boldsymbol{C}$ that transforms the direct product (22) into a fully reduced form:

$$
\left(\boldsymbol{D}^{* \mathbf{k}, m} \otimes \boldsymbol{D}^{* \mathbf{k}^{\prime}, m^{\prime}}\right) \boldsymbol{C}=\boldsymbol{C}\left[\oplus \boldsymbol{E}\left(* \mathbf{k} m, * \mathbf{k}^{\prime} m^{\prime} \mid * \mathbf{k}^{\prime \prime} m^{\prime \prime}\right) \otimes \boldsymbol{D}^{* \mathbf{k}^{\prime \prime}, m^{\prime \prime}}\right] .
$$

Here the direct sum is over all stars $* \mathbf{k}^{\prime \prime}$ and over all allowable irreps $\boldsymbol{D}^{* \mathbf{k}^{\prime \prime}, m^{\prime \prime}}$ which may arise for a given $* \mathbf{k}^{\prime \prime}$. The identity matrix $\boldsymbol{E}(l)$ is of dimension $l$.

Apart from the ordinary Kronecker products, the reductions of symmetrized (Kronecker) squares and cubes of irreps are also of considerable interest especially in applications related to the Landau theory of second-order or continuous phase transitions in crystalline solids. The theory sets necessary conditions for a continuous transition which involves evaluation of the symmetrized cubes and antisymmetrized squares of certain space-group representations [see e.g. Cracknell (1974) for details and references]. The Kronecker powers of representations are defined in exactly the same way as ordinary products. The Kronecker square of an irrep $\boldsymbol{D}^{* \mathbf{k}, m}$ is a reducible representation of dimension $\left(s \times r_{m}\right)^{2}$. In the following, we consider the decomposition of the symmetrized Kronecker square of an irrep:

$$
\left[\boldsymbol{D}^{* \mathbf{k}, m}\right]_{(2)} \sim \oplus_{* \mathbf{k}^{\prime \prime}, m^{\prime \prime}} \boldsymbol{E}\left[\left([* \mathbf{k}, m]_{(2)} \mid * \mathbf{k}^{\prime \prime}, m^{\prime \prime}\right) \otimes \boldsymbol{D}^{* \mathbf{k}^{\prime \prime}, m^{\prime \prime}}\right] .
$$

If the dimension of $\boldsymbol{D}^{* \mathbf{k}, m}$ is $d$, then the dimension of its symmetrized square is $\frac{1}{2} d(d+1)$.

The theory of the decomposition of Kronecker products and the related reduction coefficients has been developed during the second half of the last century. Details on the different approaches and references to the numerous contri-

\footnotetext{
${ }^{3}$ Here $s\left(s^{\prime}\right)$ give the number of arms of $* \mathbf{k}\left(* \mathbf{k}^{\prime}\right), r_{m}\left(r_{m^{\prime}}\right)$ stand for the dimension of the little-group irreps $\boldsymbol{D}^{\mathbf{k}, m}\left(\boldsymbol{D}^{\mathbf{k}^{\prime}, m^{\prime}}\right)$. The products $\left(s \times r_{m}\right)$ and $\left(s^{\prime} \times r_{m^{\prime}}^{\prime}\right)$ equal the dimensions of the full-group irreps $d^{* \mathbf{k}, m}=\operatorname{dim}\left(\boldsymbol{D}^{* \mathbf{k}, m}\right)$ and $d^{* \mathbf{k}^{\prime}, m^{\prime}} \stackrel{\operatorname{mim}}{=}\left(\boldsymbol{D}^{* \mathbf{k}^{\prime}, m^{\prime}}\right)$.
}

butions to that field can be found e.g. in Bradley \& Cracknell (1972) or CDML. Applying the so-called full-group method, ${ }^{4}$ Birman (1962) published the first extensive reductions for the space groups of the diamond and zinc-blende structures. The full-group method was the only one available until the work by Bradley \& Davies (1970) who developed the basis of the subgroup methods for the reduction problem.

The work involved in the construction of Kronecker products tables for space groups is rather tedious and requires considerable expertise in some aspects of the representation theory of space groups. Probably this is the reason that the only systematic and relatively complete compilations of Kronecker product tables for the space groups are given in Volumes 2, 3 and 4 of the Kronecker Product Tables (Davies \& Cracknell, 1979; Cracknell \& Davies, 1979; Davies \& Cracknell, 1980). The reductions of ordinary Kronecker products of the irreps of the special wavevectors in the representation domains of all 230 space groups are given in Volumes 2 and 3 of the series. In Volume 4, the reductions of the symmetrized squares and cubes of the special wavevectors are listed. The tables are computer produced and the method for their calculation is based on the subgroup method. However, the Kronecker Product Tables have become a bibliographic collector's item. In addition, for certain applications, the published data are not sufficient and/or it is necessary to have the data in an electronic form. The program DIRPRO carries out the reductions of ordinary Kronecker products and symmetrized squares of space-group irreps for any wavevector (inside or outside the representation domain). The program calculates the related wavevector selection rules (see below) and the reduction coefficients. The program DIRPRO is designed to compute also the Clebsch-Gordan coefficients. For the moment, this option is not included in the implemented version of DIRPRO on the Bilbao Crystallographic Server.

\subsection{The method}

For the decomposition of the Kronecker product of two space-group irreps and the determination of the corresponding reduction coefficients, we use a modification of the full-group method [cf. Birman (1974) for details and a mathematical background of the approach]. The reduction procedure applied in DIRPRO is rather similar to the one used for the solution of the subduction problem ( $\$ 3)$. The reason is obvious: in both cases, it is necessary to decompose a reducible representation into irreducible constituents. The essential differences concern the construction of the reducible representation.

The determination of the reduction coefficients of an ordinary Kronecker product (22) and of a symmetrized Kronecker square (24) of space-group irreps is carried out in two steps.

\footnotetext{
${ }^{4}$ The full-group methods deal with the full space group $\mathcal{G}$ and its irreps $\boldsymbol{D}^{* \mathbf{k}, m}$ and uses these in all stages of the reduction procedure. In contrast, the subgroup methods focus mainly on the little groups $\mathcal{G}^{\mathbf{k}}$ and its allowable irreps $\boldsymbol{D}^{\mathbf{k}, m}$. Since $\boldsymbol{D}^{* \mathbf{k}, m}$ are constructed by induction from $\boldsymbol{D}^{\mathbf{k}, m}$, it is possible to obtain an equivalent set of results of the Kronecker product reduction.
} 
4.2.1. Wavevector selection rules. The first step in the reduction procedure is the determination of the wavevector stars $* \mathbf{k}^{\prime \prime}$ that occur in the splitting of the direct product $* \mathbf{k} \otimes * \mathbf{k}^{\prime}$. One defines $* \mathbf{k}$ reduction coefficients $\left(* \mathbf{k} * \mathbf{k}^{\prime} \mid * \mathbf{k}^{\prime \prime}\right)$ for the formal description of the splitting:

$$
* \mathbf{k} \otimes * \mathbf{k}^{\prime}=\sum_{* \mathbf{k}^{\prime \prime}}\left(* \mathbf{k} * \mathbf{k}^{\prime} \mid * \mathbf{k}^{\prime \prime}\right) * \mathbf{k}^{\prime \prime} .
$$

The fact that the $s_{\mathbf{k}} s_{\mathbf{k}^{\prime}}^{\prime}$ arms of the direct product $* \mathbf{k} \otimes * \mathbf{k}^{\prime}$ must be expressible in terms of entire stars results in the following relation for $\left(* \mathbf{k} * \mathbf{k}^{\prime} \mid * \mathbf{k}^{\prime \prime}\right)$ :

$$
s_{\mathbf{k}} s_{\mathbf{k}^{\prime}}^{\prime}=\sum_{* \mathbf{k}^{\prime \prime}}\left(* \mathbf{k} * \mathbf{k}^{\prime} \mid * \mathbf{k}^{\prime \prime}\right) s_{\mathbf{k}^{\prime \prime}}^{\prime \prime}
$$

The analogous relations for the $* \mathbf{k}$ reduction coefficients for the symmetrized Kronecker product $\left([* \mathbf{k}]_{(2)} \mid * \mathbf{k}^{\prime \prime}\right)$ are of the form

$$
\begin{aligned}
{[* \mathbf{k}]_{(2)} } & =\sum_{* \mathbf{k}^{\prime \prime}}\left([* \mathbf{k}]_{(2)} \mid * \mathbf{k}^{\prime \prime}\right) * \mathbf{k}^{\prime \prime} \\
\frac{1}{2} s_{\mathbf{k}}\left(s_{\mathbf{k}}+1\right) & =\sum_{* \mathbf{k}^{\prime \prime}}\left([* \mathbf{k}]_{(2)} \mid * \mathbf{k}^{\prime \prime}\right) s_{\mathbf{k}^{\prime \prime}}^{\prime \prime}
\end{aligned}
$$

The $* \mathbf{k}$ reduction coefficients are integers and are determined by direct inspection.

4.2.2. Reduction coefficients. The number $N$ of unknown reduction coefficients equals the sum over the numbers of allowed irreps for each of the stars $* \mathbf{k}^{\prime \prime}$ that appear in the splitting of the Kronecker product. The method of linear equations is used for the determination of the reduction coefficients. The first $q$ equations follow from the dimensionality conservation conditions for each of the resultant wavevector $\operatorname{stars} * \mathbf{k}_{i}^{\prime \prime}, i=1, \ldots, q$ :

$$
\begin{gathered}
\sum_{m^{\prime \prime}}\left(* \mathbf{k} m, * \mathbf{k}^{\prime} m^{\prime} \mid * \mathbf{k}^{\prime \prime} m^{\prime \prime}\right) d^{* \mathbf{k}^{\prime \prime}, m^{\prime \prime}} \\
=r_{\mathbf{k} m} r_{\mathbf{k}^{\prime} m^{\prime}}^{\prime}\left(* \mathbf{k} * \mathbf{k}^{\prime} \mid * \mathbf{k}^{\prime \prime}\right) s_{\mathbf{k}^{\prime \prime}}^{\prime \prime}
\end{gathered}
$$

Here $d^{* \mathbf{k}^{\prime \prime}, m^{\prime \prime}}$ is the dimension of $\boldsymbol{D}^{* \mathbf{k}^{\prime \prime}, m^{\prime \prime}}$, and $r_{\mathbf{k} m}$ and $r_{\mathbf{k}^{\prime} m^{\prime}}^{\prime}$ are the dimensions of the little-group irreps of the factors $\boldsymbol{D}^{* \mathbf{k}, m}$ and $\boldsymbol{D}^{* \mathbf{k}^{\prime}, m^{\prime}}$.

The rest of the equations for the reduction coefficients are obtained from the defining relation (22) rewritten for the characters of $\boldsymbol{D}^{* \mathbf{k}, m} \otimes \boldsymbol{D}^{* \mathbf{k}^{\prime}, m^{\prime}}$ and the resultant irreps:

$$
\chi^{(* \mathbf{k}, m) \otimes\left(* \mathbf{k}^{\prime}, m^{\prime}\right)}(\mathbf{g})=\sum\left(* \mathbf{k} m, * \mathbf{k}^{\prime} m^{\prime} \mid * \mathbf{k}^{\prime \prime} m^{\prime \prime}\right) \chi^{* \mathbf{k}^{\prime \prime}, m^{\prime \prime}}(\mathbf{g}),
$$

where $\chi^{(* \mathbf{k}, m) \otimes\left(* \mathbf{k}^{\prime}, m^{\prime}\right)}(\mathbf{g})=\chi^{* \mathbf{k}, m}(\mathbf{g}) \chi^{* \mathbf{k}^{\prime}, m^{\prime}}(\mathbf{g}), \quad \mathbf{g} \in \mathcal{G}$.

Similarly, for the case of symmetrized Kronecker products, one gets the following equations:

$$
\begin{aligned}
\sum_{m^{\prime \prime}}\left([* \mathbf{k} m]_{(2)} \mid * \mathbf{k}^{\prime \prime} m^{\prime \prime}\right) d^{* \mathbf{k}^{\prime \prime}, m^{\prime \prime}} & \\
& =\frac{1}{2} r_{\mathbf{k} m}\left(r_{\mathbf{k} m}+1\right)\left(\left[* \mathbf{k}_{(2)}\right] \mid * \mathbf{k}^{\prime \prime}\right) s_{\mathbf{k}^{\prime \prime}}^{\prime \prime}, \\
\chi^{[* \mathbf{k}, m]_{(2)}}(\mathbf{g}) & =\sum\left([* \mathbf{k} m]_{(2)} \mid * \mathbf{k}^{\prime \prime} m^{\prime \prime}\right) \chi^{* \mathbf{k}^{\prime \prime}, m^{\prime \prime}}(\mathbf{g}), \\
\text { with } \chi^{[* \mathbf{k}, m]_{(2)}}(\mathbf{g}) & =\frac{1}{2}\left[\left(\chi^{* \mathbf{k}, m}(\mathbf{g})\right)^{2}+\left(\chi^{* \mathbf{k}, m}(\mathbf{g})^{2}\right)\right], \quad \mathbf{g} \in \mathcal{G} .
\end{aligned}
$$

Trial-and-error methods are used for the selection of the necessary $(N-q)$ independent linear equations for the calculation of the reduction coefficients. The search is carried out among equations of the type (30) or (32) taken for different elements of $\mathcal{G}$. A Gauss elimination procedure is used for the solution of the system of linear equations for the reduction coefficients.

\subsection{The program DIRPRO}

The input data of DIRPRO include the specification of the space group $\mathcal{G}$ by its ITA number and the data for the wavevectors. The k-vector coefficients could be referred to the primitive bases of reciprocal space (as found in CDML), to the centered dual basis or to specify the wavevector by the adjusted coefficients ( $c f$. $\$ 2.1 .3$ ). As an option, the program displays the full-group representations (not just the representations of the little groups) for the generators of the space group. There is also an option to consider the symmetrized Kronecker squares if $* \mathbf{k}=* \mathbf{k}^{\prime}$.

The structure of the output of DIRPRO follows that of $C O R R E L$. The first block lists the information about the space group $\mathcal{G}$ including its space-group number and lattice type, generators and translational coset representatives $(\boldsymbol{W}, \boldsymbol{w})$ given in $(3 \times 4)$ matrix form. The $\mathbf{k}$ vectors are specified by their input coefficients and those referred to the dual basis of the default setting of $\mathcal{G}$. The arms of the wavevector stars $* \mathbf{k}$ and $* \mathbf{k}^{\prime}$ are also shown. The wavevector selection rules are displayed in the block with the heading 'INFORMATION FOR THE SPLITTING'. Each of the resulting $* \mathbf{k}^{\prime \prime}$ stars is specified by its $\mathbf{k}$-vector coefficients referred to the dual basis of $\mathcal{G}$. Next follows the block containing information on the representations of the space group $\mathcal{G}$ for $* \mathbf{k}, * \mathbf{k}^{\prime}$ and all $* \mathbf{k}^{\prime \prime}$ stars that appear in the splitting of the direct product. The data consist of the corresponding little groups, the allowed littlegroup irreps, the chosen coset representatives of the decomposition of the group with respect to the little group and the full space-group irreps given for the generators (optional). The last block of the output, called 'REDUCTION PROBLEM', shows the decompositions of all possible direct products $\boldsymbol{D}^{* \mathbf{k}, m} \otimes \boldsymbol{D}^{* \mathbf{k}^{\prime}, m^{\prime}}$ (for each allowed $m$ and $m^{\prime}$ ) into direct sums of irreducible constituents. The reduction coefficients of the symmetrized squares are displayed optionally.

URL of the program: http://www.cryst.ehu.es/rep/ dirpro.html.

\section{Illustrative examples}

The databases and computer packages on space- and pointgroup representations form the set of basic modules that is used further in different programs on the Bilbao Crystallographic Server for applications of representation theory to specific problems of solid-state physics and chemistry. For example, symmetry-mode analysis of atomic displacements of crystalline solids could be very helpful for the proper interpretation of infrared and Raman experimental results. The program $S A M$ computes the symmetry-adapted modes at the $\Gamma$ point and studies their infrared and Raman activity. The program NEUTRON (Kirov et al., 2003) computes the phonon selection rules applicable in inelastic neutron-scattering experiments. The software package SYMMODES (Capillas et 
al., 2003) performs a group-theoretical analysis of structural phase transitions. These application programs are a subject of a forthcoming article. In the following, we illustrate the usefulness of the group-subgroup computer tools available on the Bilbao Crystallographic Server [program SUBGROUP$G R A P H$, for more details see e.g. Aroyo et al. (2006)] and the correlation relations obtained by CORREL in treating phasetransition problems.

Consider a continuous or quasi-continuous phase transition between two crystalline phases whose symmetry groups are group-subgroup related $\mathcal{G}>\mathcal{H}$. The order parameter that drives the transition (i.e. it is related to the symmetry-breaking distortion with respect to the high-symmetry phase) is known as the primary order parameter and is associated with an irrep $\boldsymbol{D}^{* \mathbf{k}, m}$ of $\mathcal{G}$, the so-called active irrep. The direction of the order parameter below the transition determines the space-group symmetry $\mathcal{H}$ which is also known as the isotropy subgroup of the order parameter. A subgroup $\mathcal{H}$ is an isotropy subgroup of $\boldsymbol{D}^{* \mathbf{k}, m}$ of $\mathcal{G}$ if and only if: (i) the subduction multiplicity of the identity irrep of $\mathcal{H}$ in $\boldsymbol{D}^{* \mathbf{k}, m} \downarrow \mathcal{H}$ is non-zero, and (ii) there exists no supergroup $\mathcal{Z}$ of $\mathcal{H}$ in the group-subgroup graph of maximal subgroups of $\mathcal{G}>\mathcal{H}$ with the same subduction multiplicity of the identity irrep of $\mathcal{Z}$ in $\boldsymbol{D}^{* \mathbf{k}, m} \downarrow \mathcal{Z}$. These conditions are known as subduction and chain-subduction criteria (see e.g. Birman, 1978). In the analysis of the symmetry break $\mathcal{G} \rightarrow \mathcal{H}$, one should consider all possible distortions compatible with the symmetry $\mathcal{H}$. Some distortions are related to order parameters associated with irreps different from that of the primary order parameter and are known as secondary order parameters. Their isotropy subgroups are in general supergroups of the space group of the low-symmetry phase in the group-subgroup graph $\mathcal{G}>\mathcal{H}$. The symmetry analysis of the primary and secondary order parameters of a transformation between a high- and a lowsymmetry phase of given space-group symmetries is known as the inverse Landau problem (Ascher \& Kobayashi, 1977). The following two 'classical' examples demonstrate how the results of the programs SUBGROUPGRAPH and CORREL can be applied for the solution of the inverse Landau problem.

\section{Example 1}

The crystal structure of $\mathrm{BaTiO}_{3}$ is of perovskite type. Above $493 \mathrm{~K}, \mathrm{BaTiO}_{3}$ has the ideal paraelectric cubic phase $(P m \overline{3} m$, No. 221). As the temperature is lowered, $\mathrm{BaTiO}_{3}$ assumes tetragonal, then orthorhombic and finally trigonal structures with slightly deformed unit cells. The three structures are ferroelectric with different directions of the spontaneous polarization axes. The possible transformation matrices for the symmetry break $P m \overline{3} m>P 4 m m$ of index 6 are listed by the program SUBGROUPGRAPH. The three different P4mm subgroups of $P m \overline{3} m$ corresponding to the three domain orientations of the tetragonal phase are conjugated in $P m \overline{3} m$. Here, we consider the case of the identity transformation matrix between the conventional bases of $P m \overline{3} \mathrm{~m}$ and $P 4 \mathrm{~mm}$. The low-symmetry space group $P 4 \mathrm{~mm}$ is a translationengleiche subgroup (or $t$ subgroup for short) of $P m \overline{3} m$ but it is not a maximal subgroup: the chain of maximal subgroups is of the form $P m \overline{3} m>P 4 / m m m>P 4 m m$. The correlation relations calculated by CORREL for $P m \overline{3} m>P 4 m m$ with $\mathbf{k}$ vector $\boldsymbol{\Gamma}=$ $(0,0,0)$ indicate the candidates for the irreps of $P m \overline{3} m$ associated with the possible primary and secondary order parameters. The subduction multiplicity of the identity irrep of $P 4 \mathrm{~mm}$ is equal to 1 for three different irreps of $P m \overline{3} \mathrm{~m}$ : $(* \mathbf{G M})(1)$ (which is the identity irrep), $(* \mathbf{G M})(6)$ and $(* \mathbf{G M})(9)$ (here we use the irrep notation of CORREL). The application of the chain-subduction criteria distinguishes between primary and secondary order parameters. The obvious isotropy group of $(* \mathbf{G M})(1)$ is the high-symmetry group itself and the irrep $(* \mathbf{G M})(1)$ is related to the possible volume change that would occur during the transformation. The run of CORREL for the pair $P m \overline{3} m>P 4 / m m m$ shows that the group $P 4 / \mathrm{mmm}$ is the isotropy subgroup for $(* \mathbf{G M})(6)$ and the associated secondary order parameter corresponds to the onset of tetragonal strain during the transformation. Finally, the physical distortion characterized by $(* \mathbf{G M})(9)$ can be related to the onset of non-zero polarization and be associated with the primary order parameter. The columns of the subduction matrices of the irreps $(* \mathbf{G M})(1),(* \mathbf{G M})(6)$ and $(* \mathbf{G M})(9)$ corresponding to the identity irrep of the isotropy groups indicate the directions of the order parameters in the irrep carrier spaces. One should note that the specific form of the order-parameter direction depends on the choice of the irrep matrices.

The application of the subduction and chain-subduction conditions for the symmetry break $\operatorname{Pm} \overline{3} m>R 3 m$, index 8 , shows that the primary distortions related to the onset of polarization along one of the main-diagonal directions of the cube is also associated with $(* \mathbf{G M})(9)$ but with order-parameter direction $(a, a, a)$. There are three more irreps of $P m \overline{3} m$ whose subduction multiplicities of the identity irrep of $R 3 m$ are different from zero: $(* \mathbf{G M})(1),(* \mathbf{G M})(4)$ and $(* \mathbf{G M})(8)$. Their isotropy groups $P m \overline{3} m, P \overline{4} 3 m$ and $R \overline{3} m$ are determined from the results of CORREL for the pairs $P m \overline{3} m>P \overline{4} 3 m$ and $P m \overline{3} m>R \overline{3} m$. The graph $P m \overline{3} m>R 3 m$ derived by the program SUBGROUPGRAPH (Fig. 2) shows that the isotropy subgroups $P \overline{4} 3 m$ and $R \overline{3} m$ are intermediate

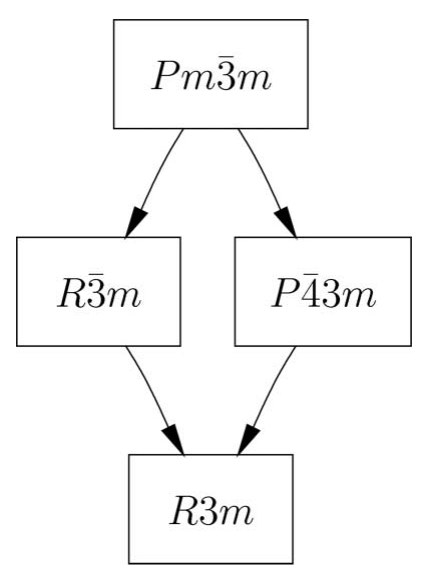

Figure 2

Graph of maximal subgroups for the group-subgroup pair $P m \overline{3} m>R 3 m$, index 8 . 
supergroups of $R 3 \mathrm{~m}$ which indicates that the corresponding order parameters are secondary ones.

\section{Example 2}

As a second example, we consider another phase transition that has been well studied both experimentally and theoretically, namely that of $\mathrm{SrTiO}_{3}$. In that case, the transformation is associated with the $\mathbf{k}$-vector point $\mathbf{R}=\left(\frac{1}{2}, \frac{1}{2}, \frac{1}{2}\right)$ which is at the border of the Brillouin zone. The high-symmetry space group is $P m \overline{3} m$ and the low-symmetry phase is tetragonal, of spacegroup symmetry $I 4 / \mathrm{mcm}$ (No. 140) with a doubling of the unit cell, i.e. $I 4 / \mathrm{mcm}$ is a general-type subgroup of $P m \overline{3} \mathrm{~m}$ of index 6. The analysis of the group-subgroup relations by SUBGROUPGRAPH shows that there are four different classes of conjugate $I 4 / \mathrm{mcm}$ subgroups. Each class consists of three different subgroups, related to the three possible doublings of the unit cell of the high-symmetry group. The different classes are distinguished by the different origin choices of the subgroup with respect to the group. For the specific transformation we are considering, $I 4 / \mathrm{mcm}$ has the lattice vectors $(\mathbf{a}-\mathbf{b}, \mathbf{a}+\mathbf{b}, 2 \mathbf{c})$ with an origin shift $\left(\frac{1}{2}, \frac{1}{2}, \frac{1}{2}\right)$ with respect to the $P m \overline{3} m$ basis. SUBGROUPGRAPH lists the following chain of maximal subgroups: $P m \overline{3} \mathrm{~m}>P 4 / \mathrm{mmm}>I 4 / \mathrm{mcm}$. The correlation relations derived by CORREL for the $* \mathbf{R}$ irreps show that only the three-dimensional irrep $(* \mathbf{R})(7)$ of $P m \overline{3} m$ subduces the identity irrep of $I 4 / \mathrm{mcm}$, with an order-parameter direction $(a, 0,0)$. Obviously, this is the irrep associated with the primary order parameter, as the only intermediate supergroup $P 4 / \mathrm{mmm}$ of $I 4 / \mathrm{mcm}$ is a $t$ subgroup of $P m \overline{3} \mathrm{~m}$. The possible secondary order parameters with $P 4 / \mathrm{mmm}$ as isotropy subgroup could be associated only with Brillouin-zone center irreps of $P m \overline{3} m$. The results of CORREL for $\boldsymbol{\Gamma}=(0,0,0)$ for the pairs $P m \overline{3} m>I 4 / m c m$ and $P m \overline{3} m>P 4 / m m m$ [tetragonal lattice vectors $(\mathbf{a}-\mathbf{b}, \mathbf{a}+\mathbf{b}, \mathbf{c})$, with no origin shift] show that the possible secondary order parameters are associated with $(* \mathbf{G M})(1)$ (volume change) and $(* \mathbf{G M})(6)$ (tetragonal distortion).

\section{Conclusions}

The Bilbao Crystallographic Server site provides a free online interface for different crystallographic databases and programs at http://www.cryst.ehu.es. The web-based working environment is divided into several shells according to different topics, from simple retrieval tools for access to crystallographic data to more sophisticated solid-state applications. The description of the databases and group-subgrouprelated tools was included in the first paper of a series of publications (Aroyo et al., 2006).

Here, we report on the group-theoretical procedures and algorithms implemented in the computer programs of the shell with some basic group-theoretical tools necessary for the numerous representation-theory applications. Information about the irreducible representations of crystallographic point groups and space groups is provided by the programs REPRES and POINT. The combination of the representation data with the crystallographic databases of the server
(Brillouin-zone database, group-subgroup data) permits one to obtain the correlations between the irreps of any pair of group-subgroup-related space groups (CORREL) which are essential in phase-transition problems. The symmetry analysis of selection rules for different phenomena involving interaction of particles or quasiparticles in a solid is based on the decomposition of Kronecker product representations into irreducible constituents. The program DIRPRO calculates the reduction for ordinary and symmetrized Kronecker products of space-group irreps.

All the programs have a user-friendly (one-button) web interface with documentation and online help. An important advantage of the programs on the server is the possibility for their internal communication. This allows the development of programs treating applications of representation theory, using as input data the output of the basic tools of the representation shell. The computer packages on the Bilbao Crystallographic Server that facilitate the study of specific problems of solid-state physics and structural chemistry are the subject of a forthcoming article.

This work has been supported by MCYT-Spain (HA2000020) and DAAD-Germany (314/AI-e-dr) within the program 'Acciones Integradas Hispano-Alemana'; DGESIC (Project No. PB98-0244) and UPV (Project No. 063.310-G19/98). Three of the authors (MA, AK and HW) gratefully acknowledge financial support by the Alexander von Humboldt foundation.

\section{References}

Altmann, S. L. \& Herzig, P. (1994). Point Group Theory Tables. Oxford: Clarendon Press.

Aroyo, M. I., Perez-Mato, J. M., Capillas, C., Kroumova, E., Ivantchev, S., Madariaga, G., Kirov, A. \& Wondratschek, H. (2006). Z. Kristallogr. 221, 15-27.

Aroyo, M. I. \& Wondratschek, H. (1995). Z. Kristallogr. 210, 243-254.

Ascher, E. \& Kobayashi, J. (1977). J. Phys. C: Solid State Phys. 10, 1349-1363.

Birman, J. L. (1962). Phys. Rev. 127, 1093-1106.

Birman, J. L. (1974). Theory of Crystal Space Groups and Infrared and Raman Lattice Processes of Insulating Crystals. In Handbuch der Physik, Bd XXV/2b, edited by S. Flüge. Berlin: Springer Verlag.

Birman, J. L. (1978). Group-Theoretical Methods in Physics, edited by P. Kramers \& A. Reickers, pp. 203-222. Berlin: Springer Verlag.

Birman, J. L. \& Berenson, R. (1974). Phys. Rev. B, 9, 4512-4517.

Bradley, C. J. \& Cracknell, A. P. (1972). The Mathematical Theory of Symmetry in Solids. Oxford: Clarendon Press.

Bradley, C. J. \& Davies, B. L. (1970). J. Math. Phys. 11, 1536-1552.

Capillas, C., Kroumova E., Perez-Mato J. M., Aroyo, M. I., Stokes, H. S. \& Hatch, D. (2003). J. Appl. Cryst. 36, 953-954.

Cracknell, A. P. (1974) Adv. Phys. 23, 673-866.

Cracknell, A. P. \& Davies, B. L. (1979). Kronecker Product Tables, Vol. 3. General Wave Vector Selection Rules and Reductions of Kronecker Products for Irreducible Representations of Triclinic, Monoclinic, Tetragonal, Trigonal and Hexagonal Space Groups. New York: IFI/Plenum. 
Cracknell, A. P., Davies, B. L., Miller, S. C. \& Love, W. F. (1979). Kronecker Product Tables, Vol. 1. General Introduction and Tables of Irreducible Representations of Space Groups. New York: IFI/ Plenum.

Davies, B. L., Cracknell, A. P. (1979). Kronecker Product Tables, Vol. 2. General Wave Vector Selection Rules and Reductions of Kronecker Products for Irreducible Representations of Orthorhombic and Cubic Space Groups. New York: IFI/Plenum.

Davies, B. L. \& Cracknell, A. P. (1980). Kronecker Product Tables, Vol. 4. General Symmetrized Powers of Irreducible Representations of Space Groups. New York: IFI/Plenum.

Hovestreydt, E. Aroyo, M. I., Sattler, S. \& Wondratschek, H. (1992). J. Appl. Cryst. 25, 544.

International Tables for Crystallography (2002). Vol. A, Space-Group Symmetry, 5th ed., edited by T. Hahn. Dordrecht: Kluwer Academic Publishers.
International Tables for Crystallography (2004). Vol. A1, Symmetry Relations between Space Groups, edited by H. Wondratschek \& U. Müller. Dordrecht: Kluwer Academic Publishers.

International Tables for Crystallography (2002). Vol. E, Subperiodic Groups, edited by V. Kopsky \& D. Litvin. Dordrecht: Kluwer Academic Publishers.

Kirov, A., Aroyo, M. I. \& Perez-Mato, J. M. (2003). J. Appl. Cryst. 36, 1085-1089.

Koster, G. F., Dimmock, J. O., Wheeler, R. G. \& Statz, H. (1963). Properties of the Thirty-two Point Groups. Cambridge: MIT Press. Kroumova, E., Aroyo, M. I., Perez-Mato, J. M., Kirov, A., Capillas, C., Ivantchev, S. \& Wondratschek, H. (2003). Phase Transit. 76, 155-170.

Mulliken, R. S. (1933). Phys. Rev. 43, 279-302.

Wintgen, G. (1941). Math. Ann. 118, 195-215.

Zak, J. (1960) J. Math. Phys. 1, 165-171. 